\title{
1 Response functions in TDDFT: concepts and implementation
}

David A. Strubbe, Lauri Lehtovaara, Angel Rubio, Miguel A. L. Marques, and Steven G. Louie

\section{David A. Strubbe}

Department of Physics, University of California, 366 LeConte Hall MC 7300, Berkeley, CA 94720-7300, USA and Materials Sciences Division, Lawrence Berkeley National Laboratory, 1 Cyclotron Road, Berkeley, CA 94720, USA dstrubbe@berkeley . edu

Lauri Lehtovaara

Laboratoire de Physique de la Matière Condensée et Nanostructures, Université Claude Bernard Lyon 1 et CNRS, 43 boulevard du 11 novembre 1918, 69622 Villeurbanne Cedex, France

lauri.lehtovaaral@iki.fi

Angel Rubio

Nano-Bio Spectroscopy Group and ETSF Scientific Development Centre, Dpto. Física de Materiales, Universidad del País Vasco, Centro de Física de Materiales CSIC-UPV/EHU-MPC and DIPC, Av. Tolosa 72, E-20018 San Sebastián, Spain and Fritz-Haber-Institut der Max-Planck-Gesellschaft, Berlin D-14195, Germany

angel.rubio@ehu.es

Miguel A. L. Marques

Laboratoire de Physique de la Matière Condensée et Nanostructures, Université Claude Bernard Lyon 1 et CNRS, 43 boulevard du 11 novembre 1918, 69622 Villeurbanne Cedex, France

miguel.marques@tddft.org

Steven G. Louie

Department of Physics, University of California, 366 LeConte Hall MC 7300, Berkeley, CA 94720-7300, USA and Materials Sciences Division, Lawrence Berkeley National Laboratory, 1 Cyclotron Road, Berkeley, CA 94720, USA sglouie@berkeley.edu

Many physical properties of interest about solids and molecules can be considered as the reaction of the system to an external perturbation, and can be expressed in terms of response functions, in time or frequency and in real or reciprocal space. Response functions in time-dependent densityfunctional theory (TDDFT) can be calculated by a variety of methods. Timepropagation is a non-perturbative approach in the time domain, whose static analogue is the method of finite differences. Other approaches are perturbative and are formulated in the frequency domain. The Sternheimer equation 
solves for the variation of the wavefunctions, the Dyson equation is used to solve directly for response functions, and the Casida equation solves for the excited states via an expansion in an electron-hole basis. These techniques can be used to study a range of different response functions, including electric, magnetic, structural, and $\boldsymbol{k} \cdot \boldsymbol{p}$ perturbations. In this chapter, we give an overview of the basic concepts behind response functions and the methods that can be employed to efficiently compute the response properties within TDDFT and the physical quantities that can be studied.

\subsection{Response function}

In this section, we will: i) show how a response function maps an external field to a physical observable, ii) discuss how a specific response function is connected to a specific physical property, iii) link the fully interacting manybody density response function with the Kohn-Sham (KS) density response function, and iv) describe how the different orders of response functions form a hierarchy.

In spectroscopic experiments, an external field $F(\boldsymbol{r}, t)$ is applied to a sample. The sample, which is a fully interacting many-electron system from the theoretical point of view, responds to the external field. Then the response can measured for some physical observable $\mathcal{P}$ :

$$
\Delta \mathcal{P}=\Delta \mathcal{P}_{F}[F]
$$

In general, the dependence of the functional $\Delta \mathcal{P}_{F}[F]$ on $F$ is very complex, as it must reproduce the response for a field of any strength and shape. However, if the external field is weak, the response can be expanded as a power series with respect to the field strength [Bernard 1959, Peterson 1967]. The first-order response, also called the linear response of the observable,

$$
\delta \mathcal{P}^{(1)}(\boldsymbol{r} ; t)=\int \mathrm{d} t^{\prime} \int \mathrm{d}^{3} r^{\prime} \chi_{\mathcal{P} \leftarrow F}^{(1)}\left(\boldsymbol{r}, \boldsymbol{r}^{\prime} ; t-t^{\prime}\right) \delta F^{(1)}\left(\boldsymbol{r}^{\prime} ; t^{\prime}\right)
$$

is a convolution of $\chi_{\mathcal{P} \leftarrow F}^{(1)}\left(\boldsymbol{r}, \boldsymbol{r}^{\prime} ; t-t^{\prime}\right)$, the linear response function, and $\delta F^{(1)}\left(\boldsymbol{r}^{\prime} ; t^{\prime}\right)$, the field expanded to first order in the field strength. The linear response function is nonlocal in space and in time, but the above time convolution simplifies to a product in frequency space:

$$
\delta \mathcal{P}^{(1)}(\boldsymbol{r} ; \omega)=\chi_{\mathcal{P} \leftarrow F}^{(1)}\left(\boldsymbol{r}, \boldsymbol{r}^{\prime} ; \omega\right) \delta F^{(1)}\left(\boldsymbol{r}^{\prime} ; \omega\right) .
$$

The linear response function $\chi_{\mathcal{P} \leftarrow F}^{(1)}\left(\boldsymbol{r}, \boldsymbol{r}^{\prime} ; \omega\right)$ depends only on a single frequency $\omega$, which is a consequence of the homogeneity of time.

At every order in the field strength, each observable/field pair has its own response function that is connected to a specific physical property. For example, the first-order response of the dipole moment to a dipole electric 
field in first order is the polarizability: $\alpha=\frac{\partial \mu}{\partial \mathcal{E}}$, the second-order response of the same pair provides the hyperpolarizability, and the first-order response of the magnetic moment to a homogeneous magnetic field is the magnetic susceptibility.

\subsubsection{Linear density response}

Perhaps the most important response function, from the TDDFT point of view, is the linear density response function $\chi_{n \leftarrow v_{\text {ext }}}^{(1)}\left(\boldsymbol{r}, \boldsymbol{r}^{\prime}, t-t^{\prime}\right)$, which gives the linear density response $\delta n^{(1)}(\boldsymbol{r}, t)$ to an external scalar potential $\delta v_{\text {ext }}\left(\boldsymbol{r}^{\prime}, t^{\prime}\right)$ :

$$
\delta n(\boldsymbol{r}, t)=\int \mathrm{d} t^{\prime} \int \mathrm{d}^{3} r^{\prime} \chi\left(\boldsymbol{r}, \boldsymbol{r}^{\prime}, t-t^{\prime}\right) \delta v_{\text {ext }}\left(\boldsymbol{r}^{\prime}, t^{\prime}\right),
$$

where $\chi\left(\boldsymbol{r}, \boldsymbol{r}^{\prime}, t-t^{\prime}\right)$ is just a shorthand notation for $\chi_{n \leftarrow v_{\text {ext }}}^{(1)}\left(\boldsymbol{r}, \boldsymbol{r}^{\prime}, t-t^{\prime}\right)$. If the density response function $\chi\left(\boldsymbol{r}, \boldsymbol{r}^{\prime}, t-t^{\prime}\right)$ is solved explicitly, it can then be used to calculate the first-order response of all properties derivable from the density with respect to any scalar field (e.g., polarizability, magnetic susceptibility).

The fully interacting many-body response function can be obtained from the corresponding Kohn-Sham system [Gross 1985, Petersilka 1996]. The Kohn-Sham system describes a non-interacting system of the electrons subject to an external potential $v_{\mathrm{KS}}(\boldsymbol{r}, t)$, which is the effective Kohn-Sham potential. Therefore, the so-called linear Kohn-Sham (density) response function measures how the density changes upon linear variation of the Kohn-Sham potential $v_{\mathrm{KS}}(\boldsymbol{r}, t)$ :

$$
\delta n(\boldsymbol{r}, t)=\int \mathrm{d} t^{\prime} \int \mathrm{d}^{3} r^{\prime} \chi_{\mathrm{KS}}\left(\boldsymbol{r}, \boldsymbol{r}^{\prime}, t-t^{\prime}\right) \delta v_{\mathrm{KS}}\left(\boldsymbol{r}^{\prime}, t^{\prime}\right) .
$$

Note that, by virtue of the KS construction, the variation of the density $\delta n(\boldsymbol{r}, t)$ is equal to the fully interacting system. In addition to the external potential $v_{\text {ext }}(\boldsymbol{r}, t)$, the effective Kohn-Sham potential has contributions from the Hartree and the exchange-correlation potentials:

$$
\delta v_{\mathrm{KS}}\left(\boldsymbol{r}^{\prime}, t^{\prime}\right)=\delta v_{\mathrm{ext}}\left(\boldsymbol{r}^{\prime}, t^{\prime}\right)+\delta v_{\mathrm{H}}[n]\left(\boldsymbol{r}^{\prime}, t^{\prime}\right)+\delta v_{\mathrm{xc}}[n]\left(\boldsymbol{r}^{\prime}, t^{\prime}\right)
$$

where

$$
\delta v_{H}[n]\left(\boldsymbol{r}^{\prime}, t^{\prime}\right)=\int \mathrm{d} t^{\prime \prime} \int \mathrm{d}^{3} r^{\prime \prime} \frac{\delta\left(t^{\prime}-t^{\prime \prime}\right)}{\left|\boldsymbol{r}^{\prime}-\boldsymbol{r}^{\prime \prime}\right|} \delta n\left(\boldsymbol{r}^{\prime \prime}, t^{\prime \prime}\right) .
$$

To calculate the variation of the exchange-correlation (XC) potential, one simply employs the chain-rule for functional derivatives:

$$
\delta v_{\mathrm{xc}}[n]\left(\boldsymbol{r}^{\prime}, t^{\prime}\right)=\int \mathrm{d}^{3} r^{\prime \prime} \int t^{\prime \prime} f_{\mathrm{xc}}\left[n_{\mathrm{GS}}\right]\left(\boldsymbol{r}^{\prime}, \boldsymbol{r}^{\prime \prime}, t-t^{\prime}\right) \delta n\left(\boldsymbol{r}^{\prime \prime}, t^{\prime \prime}\right) .
$$


The exchange-correlation kernel

$$
f_{\mathrm{xc}}\left[n_{\mathrm{GS}}\right]\left(\boldsymbol{r}^{\prime}, \boldsymbol{r}^{\prime \prime}, t-t^{\prime}\right)=\left.\frac{\delta v_{\mathrm{xc}}[n]\left(\boldsymbol{r}^{\prime}, t^{\prime}\right)}{\delta n\left(\boldsymbol{r}^{\prime \prime}, t^{\prime \prime}\right)}\right|_{n=n_{\mathrm{GS}}}
$$

is the functional derivative of the exchange-correlation potential with respect to the density at the ground-state density $n_{\mathrm{GS}}$. Note that the exchangecorrelation kernel $f_{\mathrm{xc}}\left[n_{\mathrm{GS}}\right]\left(\boldsymbol{r}^{\prime}, \boldsymbol{r}^{\prime \prime}, t-t^{\prime}\right)$ is a functional of the ground-state density and can be evaluated before any response calculation.

For the same external potential $v_{\text {ext }}(\boldsymbol{r}, t)$, the fully interacting and the Kohn-Sham density responses must be the same. Therefore, we can set the right-hand-side of Eq. (1.4) equal to the right-hand-side of Eq. (1.5), and use Eq. (1.4) once more to replace $\delta n$ in Eq. (1.8):

$$
\begin{aligned}
& \int \mathrm{d} t^{\prime} \int \mathrm{d}^{3} r^{\prime} \chi\left(\boldsymbol{r}, \boldsymbol{r}^{\prime}, t-t^{\prime}\right) \delta v_{\mathrm{ext}}\left(\boldsymbol{r}^{\prime}, t^{\prime}\right)= \\
& \int \mathrm{d} t^{\prime} \int \mathrm{d}^{3} r^{\prime} \chi_{\mathrm{KS}}\left(\boldsymbol{r}, \boldsymbol{r}^{\prime}, t-t^{\prime}\right) \delta v_{\mathrm{ext}}\left(\boldsymbol{r}^{\prime}, t^{\prime}\right)+\int \mathrm{d} t^{\prime} \int \mathrm{d}^{3} r^{\prime} \chi_{\mathrm{KS}}\left(\boldsymbol{r}, \boldsymbol{r}^{\prime}, t-t^{\prime}\right) \\
& \times \int \mathrm{d} t^{\prime \prime} \int \mathrm{d}^{3} r^{\prime \prime}\left[\frac{\delta\left(t^{\prime}-t^{\prime \prime}\right)}{\left|\boldsymbol{r}^{\prime}-\boldsymbol{r}^{\prime \prime}\right|}+f_{\mathrm{xc}}\left[n_{\mathrm{GS}}\right]\left(\boldsymbol{r}^{\prime}, \boldsymbol{r}^{\prime \prime}, t^{\prime}-t^{\prime \prime}\right)\right] \\
& \quad \times \int \mathrm{d} t^{\prime \prime \prime} \int \mathrm{d}^{3} r^{\prime \prime \prime} \chi\left(\boldsymbol{r}^{\prime \prime}, \boldsymbol{r}^{\prime \prime \prime}, t^{\prime \prime}-t^{\prime \prime \prime}\right) \delta v_{\mathrm{ext}}\left(\boldsymbol{r}^{\prime \prime \prime}, t^{\prime \prime \prime}\right)
\end{aligned}
$$

As the density response function is an intrinsic property of the system, it cannot depend on the detailed form of the external potential. The terms multiplying the external potential $v_{\text {ext }}(\boldsymbol{r}, t)$ must be point-wise equal, from which we obtain a Dyson-like equation for the density response function, which reads in frequency space:

$$
\begin{aligned}
& \chi\left(\boldsymbol{r}, \boldsymbol{r}^{\prime}, \omega\right)=\chi_{\mathrm{KS}}\left(\boldsymbol{r}, \boldsymbol{r}^{\prime}, \omega\right)+\int \mathrm{d}^{3} r^{\prime \prime} \int \mathrm{d}^{3} r^{\prime \prime \prime} \chi_{\mathrm{KS}}\left(\boldsymbol{r}, \boldsymbol{r}^{\prime \prime}, \omega\right) \\
& \quad \times\left[\frac{1}{\left|\boldsymbol{r}^{\prime \prime}-\boldsymbol{r}^{\prime \prime \prime}\right|}+f_{\mathrm{xc}}\left[n_{\mathrm{GS}}\right]\left(\boldsymbol{r}^{\prime \prime}, \boldsymbol{r}^{\prime \prime \prime}, \omega\right)\right] \chi\left(\boldsymbol{r}^{\prime \prime \prime}, \boldsymbol{r}^{\prime}, \omega\right) .
\end{aligned}
$$

The Kohn-Sham density response function $\chi_{\mathrm{KS}}(\boldsymbol{r}, \boldsymbol{r}, \omega)$ is straightforward to obtain from first-order perturbation theory and has poles at the Kohn-Sham energy differences:

$$
\begin{aligned}
& \chi_{\mathrm{KS}}\left(\boldsymbol{r}, \boldsymbol{r}^{\prime}, \omega\right)= \\
& \lim _{\eta \rightarrow 0^{+}} \sum_{a, i}\left(n_{i}-n_{a}\right)\left[\frac{\varphi_{i}^{*}(\boldsymbol{r}) \varphi_{a}(\boldsymbol{r}) \varphi_{i}\left(\boldsymbol{r}^{\prime}\right) \varphi_{a}^{*}\left(\boldsymbol{r}^{\prime}\right)}{\omega-\left(\varepsilon_{a}-\varepsilon_{i}\right)+i \eta}-\frac{\varphi_{i}(\boldsymbol{r}) \varphi_{a}^{*}(\boldsymbol{r}) \varphi_{a}\left(\boldsymbol{r}^{\prime}\right) \varphi_{i}^{*}\left(\boldsymbol{r}^{\prime}\right)}{\omega-\left(\varepsilon_{i}-\varepsilon_{a}\right)+\mathrm{i} \eta}\right],
\end{aligned}
$$


where $\varphi_{i}(\boldsymbol{r})$ and $\varphi_{a}(\boldsymbol{r})$ are occupied and unoccupied KS orbitals, respectively, and $\varepsilon_{i}$ and $\varepsilon_{a}$ are the corresponding KS eigenvalues. ${ }^{1}$ The equation (1.11) can be formally written as

$$
\chi=\left(1-\chi_{\mathrm{KS}} f_{\mathrm{Hxc}}\right)^{-1} \chi_{\mathrm{KS}},
$$

where all terms on the right-hand-side are known from a ground-state KohnSham calculation. This equation can be used directly to calculate the fully interacting density response function from a Kohn-Sham system [Hybertsen 1987].

Before introducing practical methods for calculating TDDFT response in section 1.2 , we will briefly discuss higher-order responses.

\subsubsection{Higher-order density response}

In linear response, a system interacts only once with the external field and only with the field component which is first-order with respect to the fieldstrength parameter. For example, the magnetic field changes the kineticenergy operator in the following way:

$$
\frac{1}{2}\left(\hat{p}+\frac{\lambda}{c} \hat{A}\right)^{2}=\frac{1}{2} \hat{p}^{2}+\frac{\lambda}{2 c} \hat{p} \cdot \hat{A}+\frac{\lambda}{2 c} \hat{A} \cdot \hat{p}+\frac{\lambda^{2}}{2 c^{2}} \hat{A}^{2}
$$

but only the terms $\hat{p} \cdot \hat{A}$ and $\hat{A} \cdot \hat{p}$ contribute to the linear response, because $\hat{A}^{2}$ term is second order with respect to the field strength parameter $\lambda$.

In second-order response, a system interacts twice with the linear component of the external field, but in addition, it also interacts once with the quadratic component of the external field $\delta v_{\text {ext }}^{(2)}\left(e . g ., \hat{A}^{2}\right)$ if it exists. The second-order density response equation reads

$$
\begin{aligned}
& \delta n^{(2)}(\boldsymbol{r}, t)= \\
& \frac{1}{2} \int \mathrm{d} t^{\prime} \int \mathrm{d} t^{\prime \prime} \int \mathrm{d}^{3} r^{\prime} \int \mathrm{d}^{3} r^{\prime \prime} \\
& \chi^{(2)}\left(\boldsymbol{r}, t, \boldsymbol{r}^{\prime}, t^{\prime}, \boldsymbol{r}^{\prime \prime}, t^{\prime \prime}\right) \delta v_{\text {ext }}^{(1)}\left(\boldsymbol{r}^{\prime}, t^{\prime}\right) \delta v_{\text {ext }}^{(1)}\left(\boldsymbol{r}^{\prime \prime}, t^{\prime \prime}\right) \\
& +\int \mathrm{d} t^{\prime} \int \mathrm{d}^{3} r^{\prime} \chi^{(1)}\left(\boldsymbol{r}, t, \boldsymbol{r}^{\prime}, t^{\prime}\right) \delta v_{\text {ext }}^{(2)}\left(\boldsymbol{r}^{\prime}, t^{\prime}\right) .
\end{aligned}
$$

or in frequency space

$$
\begin{aligned}
\delta n^{(2)}(\boldsymbol{r}, \omega)= & \frac{1}{2} \int \mathrm{d} \omega^{\prime} \int \mathrm{d} \omega^{\prime \prime} \int \mathrm{d}^{3} r^{\prime} \int \mathrm{d}^{3} r^{\prime \prime} \delta\left(\omega-\left(\omega^{\prime}+\omega^{\prime \prime}\right)\right) \\
\times & \times \chi^{(2)}\left(\boldsymbol{r}, \boldsymbol{r}^{\prime}, \boldsymbol{r}^{\prime \prime}, \omega, \omega^{\prime}, \omega^{\prime \prime}\right) \delta v_{\text {ext }}^{(1)}\left(\boldsymbol{r}^{\prime}, \omega^{\prime}\right) \delta v_{\text {ext }}^{(1)}\left(\boldsymbol{r}^{\prime \prime}, \omega^{\prime \prime}\right) \\
& +\int \mathrm{d}^{3} r^{\prime} \chi^{(1)}\left(\boldsymbol{r}, t, \boldsymbol{r}^{\prime}, \omega\right) \delta v_{\text {ext }}^{(2)}\left(\boldsymbol{r}^{\prime}, \omega\right),
\end{aligned}
$$

\footnotetext{
${ }^{1}$ This is the causal KS response function. The time-ordered KS response function would have - i $\eta$ in the second term.
} 
where $\delta\left(\omega-\left(\omega^{\prime}+\omega^{\prime \prime}\right)\right)$ comes from the conservation of energy. The secondorder response function $\chi^{(2)}\left(\boldsymbol{r}, \boldsymbol{r}^{\prime}, \boldsymbol{r}^{\prime \prime}, \omega, \omega^{\prime}, \omega^{\prime \prime}\right)$ mixes two frequencies (which can be different, $\omega_{1}$ and $\omega_{2}$, or the same, $\omega_{1}= \pm \omega_{2}$ ) to a new frequency. For example, if the field is monochromatic having only frequency $\omega_{1}$, the secondorder response generates second harmonics at frequency $2 \omega_{1}$, and optical rectification at frequency $\omega=\omega_{1}-\omega_{1}=0$.

The second-order Kohn-Sham response is fairly straightforward to obtain [Senatore 1987], and it reads

$$
\begin{aligned}
& \delta n^{(2)}(\boldsymbol{r}, t)= \\
& \frac{1}{2} \int \mathrm{d} t^{\prime} \int \mathrm{d} t^{\prime \prime} \int \mathrm{d}^{3} r^{\prime} \int \mathrm{d}^{3} r^{\prime \prime} \chi_{\mathrm{KS}}^{(2)}\left(\boldsymbol{r}, t, \boldsymbol{r}^{\prime}, t^{\prime}, \boldsymbol{r}^{\prime \prime}, t^{\prime \prime}\right) \delta v_{\text {ext }}^{(1)}\left(\boldsymbol{r}^{\prime}, t^{\prime}\right) \delta v_{\text {ext }}^{(1)}\left(\boldsymbol{r}^{\prime \prime}, t^{\prime \prime}\right) \\
& +\int \mathrm{d} t^{\prime} \int \mathrm{d}^{3} r^{\prime} \chi_{\mathrm{KS}}^{(1)}\left(\boldsymbol{r}, t, \boldsymbol{r}^{\prime}, t^{\prime}\right) \delta v_{\mathrm{ext}}^{(2)}\left(\boldsymbol{r}^{\prime}, t^{\prime}\right) . \\
& +\frac{1}{2} \int \mathrm{d} t^{\prime} \int \mathrm{d} t^{\prime \prime} \int \mathrm{d} t^{\prime \prime \prime} \int \mathrm{d}^{3} r^{\prime} \int \mathrm{d}^{3} r^{\prime \prime} \int \mathrm{d}^{3} r^{\prime \prime \prime} \chi_{\mathrm{KS}}^{(1)}\left(\boldsymbol{r}, t, \boldsymbol{r}^{\prime}, t^{\prime}\right) \\
& \times M_{\mathrm{xc}}\left(\boldsymbol{r}^{\prime}, t^{\prime}, \boldsymbol{r}^{\prime \prime}, t^{\prime \prime}, \boldsymbol{r}^{\prime \prime \prime}, t^{\prime \prime \prime}\right) \delta n^{(1)}\left(\boldsymbol{r}^{\prime \prime}, t^{\prime \prime}\right) \delta n^{(1)}\left(\boldsymbol{r}^{\prime \prime \prime}, t^{\prime \prime \prime}\right) \\
& +\int \mathrm{d} t^{\prime} \int \mathrm{d} t^{\prime \prime} \int \mathrm{d}^{3} r^{\prime} \int \mathrm{d}^{3} r^{\prime \prime} \chi_{\mathrm{KS}}^{(1)}\left(\boldsymbol{r}, t, \boldsymbol{r}^{\prime}, t^{\prime}\right) \\
& \quad \times\left(\frac{\delta\left(t^{\prime}-t^{\prime \prime}\right)}{\left|\boldsymbol{r}^{\prime}-\boldsymbol{r}^{\prime \prime}\right|}+f_{\mathrm{xc}}\left(\boldsymbol{r}^{\prime}, t^{\prime}, \boldsymbol{r}^{\prime \prime}, t^{\prime \prime}\right)\right) \delta n^{(2)}\left(\boldsymbol{r}^{\prime \prime}, t^{\prime \prime}\right),
\end{aligned}
$$

where

$$
M_{\mathrm{xc}}\left(\boldsymbol{r}^{\prime}, t^{\prime}, \boldsymbol{r}^{\prime \prime}, t^{\prime \prime}, \boldsymbol{r}^{\prime \prime \prime}, t^{\prime \prime \prime}\right)=\left.\frac{\delta^{2} v_{\mathrm{xc}}\left(\boldsymbol{r}^{\prime}, t^{\prime}\right)}{\delta n\left(\boldsymbol{r}^{\prime \prime}, t^{\prime \prime}\right) \delta n\left(\boldsymbol{r}^{\prime \prime \prime}, t^{\prime \prime \prime}\right)}\right|_{n=n_{\mathrm{GS}}}
$$

If $\chi^{(1)}$ is already solved for, the 2nd-order response equation can then be obtained by combining Eqs. (1.4), (1.17) and (1.15).

Higher-order responses are also straightforward to construct but become quickly cumbersome. The response equations form a hierarchic structure where the $i$ th-order response requires the $(i-1)$ th- and lower-order responses. Note that all functional derivatives of the XC potentials are with respect to the ground-state density.

\subsection{Methods for calculating response functions}

In this section, we will briefly describe three different methods to calculate response from TDDFT: i) time-propagation, ii) Sternheimer, and iii) Casida method. The time-propagation method [Yabana 1999] simply propagates a system under a given external field. The density response is obtained directly as the difference between the time-dependent density and the ground-state state density. As the method is nonperturbative, all orders of response are 
included in the calculation, and therefore, specific orders must be numerically extracted. The Sternheimer method [Sternheimer 1954, Baroni 1987b, Gonze 1995b, Andrade 2007] solves for a specific order of the response for a specific field in frequency space (i.e, it is a perturbative approach). The Sternheimer equations form a hierarchic structure, where higher-order responses can be calculated from lower-order responses. The Casida method [Casida 1995], instead of finding the response, finds the poles and residues of the first-order response function, which corresponds to finding the resonant transitions of a system. Note that physically all these techniques are equivalent as they are all based on Kohn-Sham DFT and are simply different ways to obtain the same quantities. Often the choice between them is done purely on numerical arguments, as each one is more adapted to certain numerical implementations [Marques 2006].

We will show that the three methods are connected to each other in a simple way. Since the purpose is to connect all these approaches, we will consider only weak perturbations. For pedagogical reasons, we make the following simplifying assumptions in the discussion below that can be easily generalized (see discussion at the end of this Section): i) the exchange-correlation functional does not have memory, i.e., we work within the adiabatic approximation (see ), ii) the system is spin-unpolarized, iii) we have no fractional occupations, and iv) we use no $k$-point sampling, i.e., only $\Gamma$-point or a nonperiodic system, which allows us to use purely real $(i . e$. , imaginary part is zero) ground-state Kohn-Sham wavefunctions. Assumption (i) is in practice not very restrictive, as a large majority of the functionals used in TDDFT are, indeed, adiabatic. Assumptions (ii)-(iv) are valid, for example, for closedshell molecules. In any case it is fairly straightforward (but cumbersome) to remove the assumptions from the derivation. We will return to this topic in the end of the section.

\subsubsection{Time-propagation method}

In the time-propagation approach, the time-dependent Kohn-Sham equations are propagated in real-time (for details, see ), i.e., by solving the following nonlinear partial differential equation

$$
\mathrm{i} \frac{\partial}{\partial t} \varphi_{k}(\boldsymbol{r}, t)=\hat{H}_{\mathrm{KS}}\left[n\left(\boldsymbol{r}^{\prime}, t\right)\right](\boldsymbol{r}, t) \varphi_{k}(\boldsymbol{r}, t),
$$

starting from time $t=0$ with the initial condition $\psi_{k}(\boldsymbol{r}, t=0)=\phi_{k}^{(0)}(\boldsymbol{r})$, where $\phi_{k}^{(0)}(\boldsymbol{r})$ are the ground-state Kohn-Sham wavefunctions. Here, we already have applied the adiabatic approximation by assuming that $\hat{H}_{\mathrm{KS}}[n](\boldsymbol{r}, t)$ has a functional dependence only on the instantaneous density $n(\boldsymbol{r}, t)$ instead of on its whole history.

If no perturbation is applied to the system, the system remains in the ground-state and the time-evolution of the KS wavefunctions is trivial: $\psi_{k}(t)=$

Cross Chap. TPTDDFT
Cross Chap. Adiabatic approximation 
$\phi^{(0)} \mathrm{e}^{-\mathrm{i} \varepsilon_{k}^{(0)} t}$. If we apply a weak time-dependent external perturbation with a given frequency $\omega$, the time-evolution becomes nontrivial because of the nonlinearity of the Kohn-Sham Hamiltonian. A general form for a weak timedependent external perturbation with a given frequency $\omega$ is

$$
v_{\text {ext }}(\boldsymbol{r}, t)=\lambda v_{\text {ext }}^{\cos }(\boldsymbol{r}) \cos (\omega t)+\lambda v_{\text {ext }}^{\sin }(\boldsymbol{r}) \sin (\omega t)
$$

or, rewriting in terms of the exponential

$$
v_{\text {ext }}(\boldsymbol{r}, t)=\lambda v_{\text {ext }}^{+\omega}(\boldsymbol{r}) \mathrm{e}^{+i \omega t}+\lambda v_{\text {ext }}^{-\omega}(\boldsymbol{r}) \mathrm{e}^{-i \omega t},
$$

where $\lambda$ is the strength of the perturbation. If we now insert this external potential to the TDKS equation and propagate in time, we can obtain physical observables from the time-dependent expectation values. For example, if we apply a weak delta pulse of a dipole electric field ${ }^{2}$

$$
v_{\mathrm{ext}}(\boldsymbol{r}, t)=-e \boldsymbol{r} \cdot \boldsymbol{K} \delta(t)=-e \boldsymbol{r} \cdot \boldsymbol{K} \frac{1}{2 \pi} \int_{-\infty}^{\infty} \mathrm{d} \omega \exp (\mathrm{i} \omega t),
$$

we simply replace the ground-state wavefunctions (solutions to Hamiltonian $\left.\hat{H}_{\mathrm{KS}}^{(0)}\right)$ by

$$
\begin{array}{r}
\psi_{k}\left(\boldsymbol{r}, t=0^{+}\right)=\exp \left\{-\mathrm{i} \int_{0^{-}}^{0+} \mathrm{d} t\left[\hat{H}_{\mathrm{KS}}^{(0)}(t)-e \boldsymbol{r} \cdot \boldsymbol{K} \delta(t)\right]\right\} \psi_{k}\left(\boldsymbol{r}, t=0^{-}\right) \\
=\exp (\mathrm{i} e \boldsymbol{r} \cdot \boldsymbol{K}) \psi_{k}\left(\boldsymbol{r}, t=0^{-}\right), \quad(1
\end{array}
$$

and propagate the free oscillations in time. Then the time-dependent dipole moment

$$
\boldsymbol{\mu}(t)=-e \int \mathrm{d}^{3} r \boldsymbol{r} n(\boldsymbol{r}, t)
$$

can be used to extract the dynamic polarizability tensor $\alpha(\omega)$. The series expansion of the time-dependent dipole moment in frequency space reads

$$
\mu_{\gamma}(\omega)=\mu_{\gamma}(0)+\alpha_{\gamma \delta}(\omega) K_{\delta}+\mathcal{O}\left(K_{\delta}^{2}\right)
$$

where $\mu_{\gamma}(\omega)$ is the Fourier transform of the time-dependent dipole moment in direction $\gamma$, and $\mu_{\gamma}(0)$ is the static dipole moment. We obtain ${ }^{3}$

$$
\alpha_{\gamma \delta}(\omega)=\frac{1}{K_{\delta}} \int_{0}^{\infty} \mathrm{d} t\left[\mu_{\gamma}(t)-\mu_{\gamma}\left(0^{-}\right)\right] \mathrm{e}^{-\mathrm{i} \omega t}+\mathcal{O}\left(K_{\delta}\right) .
$$

The imaginary part of the diagonal component of the dynamic polarizability $\mathcal{I}\left[\alpha_{\delta \delta}(\omega)\right]$ is proportional to the absorption spectrum. The above equation

$\overline{{ }^{2}}$ Note that $\boldsymbol{K}$ has units of electric field times time.

${ }^{3}$ Note that the integration begins from $t=0^{-}$instead of $-\infty$, which basically corresponds to adding a Heaviside function $\theta\left(t-0^{-}\right)$inside the Fourier transform. 
includes an integral over infinite time. Obviously, infinite time-propagation is not possible in practice, and we have to add an artificial lifetime to the equation by introducing a decay $\mathrm{e}^{-\eta t}$ :

$$
\alpha_{\gamma \delta}(\omega)=\frac{1}{K_{\delta}} \int_{0}^{\infty} \mathrm{d} t\left[\mu_{\gamma}(t)-\mu_{\gamma}\left(0^{-}\right)\right] \mathrm{e}^{-\mathrm{i} \omega t} \mathrm{e}^{-\eta t}+\mathcal{O}\left(K_{\delta}\right)
$$

which corresponds to forcing all excitations to decay back to the ground state with rate $\eta$.

Higher-order responses (e.g., hyperpolarizibilities) are automatically considered in the calculation. However, if the field is chosen weak enough, they have negligible contribution, as should be the case for a linear-response calculation. If the perturbation strength is increased, the nonlinear contributions begin to increase: 2 nd order quadratically, 3rd order cubically, etc. In addition to the different scaling with respect to the perturbation strength, higher-order responses appear at combinations of existing frequencies, which will be discussed in the next subsection. To disentangle the different contributions from the time propagation scheme is not always a well-defined procedure.

\subsubsection{Sternheimer method}

The time-propagation approach propagates the TDKS equations in real-time. If we apply time-dependent perturbation theory and transform the equations to frequency space, we obtain the Sternheimer method, which is also known as density-functional perturbation theory (DFPT), particularly in its static form [Baroni 2001], and as "coupled perturbed Kohn-Sham" (CPKS) in the quantum-chemistry literature.

As the time-dependent external perturbation was chosen weak, we can expand the TD-KS states and the TD-KS-Hamiltonian as a power series with respect to the perturbation strength $\lambda$. The perturbation expansion ${ }^{4}$ of the TD-KS states reads

$$
\varphi_{k}(\boldsymbol{r}, t)=\varphi_{k}^{(0)}(\boldsymbol{r}, t)+\lambda \varphi_{k}^{(1)}(\boldsymbol{r}, t)+\lambda^{2} \varphi_{k}^{(2)}(\boldsymbol{r}, t)+\ldots
$$

where the zeroth-order response has trivial time-dependence $\varphi_{k}^{(0)}(\boldsymbol{r}, t)=$ $\varphi_{k}^{(0)}(\boldsymbol{r}) \mathrm{e}^{-i \varepsilon_{k}^{(0)} t}$. The perturbation expansion of the TD-KS Hamiltonian reads

$$
\begin{aligned}
\hat{H}_{\mathrm{KS}}(\boldsymbol{r}, t)=\hat{H}_{\mathrm{KS}}^{(0)}\left[n^{(0)}\left(\boldsymbol{r}^{\prime}, t\right)\right](\boldsymbol{r}) & \\
& +\lambda v_{\mathrm{ext}}^{(1)}(\boldsymbol{r}, t)+\lambda^{2} v_{\mathrm{ext}}^{(2)}(\boldsymbol{r}, t)+\ldots \\
& +\lambda \hat{H}_{\mathrm{KS}}^{(1)}\left[n\left(\boldsymbol{r}^{\prime}, t\right)\right](\boldsymbol{r}, t)+\lambda^{2} \hat{H}_{\mathrm{KS}}^{(2)}\left[n\left(\boldsymbol{r}^{\prime}, t\right)\right](\boldsymbol{r}, t)+\ldots
\end{aligned}
$$

\footnotetext{
${ }^{4}$ Note that this expansion is not a Taylor expansion.
} 
where $\hat{H}_{\mathrm{KS}}^{(0)}\left[n^{(0)}\left(\boldsymbol{r}^{\prime}\right)\right](\boldsymbol{r})$ is the ground-state Hamiltonian. The $\hat{H}_{\mathrm{KS}}^{(k)}\left[n\left(\boldsymbol{r}^{\prime}, t\right)\right](\boldsymbol{r}, t)$ are the $k$ th-order response Hamiltonians, i.e. $k$ th derivatives of the Hamiltonian with respect to the magnitude of the bare external perturbation. ${ }^{5}$ These response Hamiltonians arise from the nonlinearity of the TD-KS Hamiltonian, when the system is perturbed by the time-dependent external potential $v_{\text {ext }}(\boldsymbol{r}, t)$.

The response Hamiltonians require the time-dependent density

$$
\begin{aligned}
& n(\boldsymbol{r}, t)=\sum_{k} n_{k}\left|\varphi_{k}(\boldsymbol{r}, t)\right|^{2} \\
& =\sum_{k} n_{k}\left\{\left|\varphi_{k}^{(0)}(\boldsymbol{r}, t)\right|^{2}+\lambda\left[\left(\varphi_{k}^{(0)}(\boldsymbol{r}, t)\right)^{*} \varphi^{(1)}(\boldsymbol{r}, t)+\left(\varphi_{k}^{(1)}(\boldsymbol{r}, t)\right)^{*} \varphi^{(0)}(\boldsymbol{r}, t)\right]\right. \\
& \left.+\lambda^{2}\left[\left(\varphi_{k}^{(2)}(\boldsymbol{r}, t)\right)^{*} \varphi^{(0)}(\boldsymbol{r}, t)+\left(\varphi_{k}^{(0)}(\boldsymbol{r}, t)\right)^{*} \varphi^{(2)}(\boldsymbol{r}, t)+\left|\varphi_{k}^{(1)}(\boldsymbol{r}, t)\right|^{2}\right]+\ldots\right\} \\
& =n^{(0)}(\boldsymbol{r}, t)+\lambda n^{(1)}(\boldsymbol{r}, t)+\lambda^{2} n^{(2)}(\boldsymbol{r}, t)+\ldots
\end{aligned}
$$

where $n_{k}$ is the occupation of the $k$ th KS-state.

Each response Hamiltonian $\hat{H}_{\mathrm{KS}}^{(k)}\left[n\left(\boldsymbol{r}^{\prime}, t\right)\right](\boldsymbol{r}, t)$ depends only on the response densities $n^{(j \leq k)}(\boldsymbol{r}, t)$ which are of lower or equal order. For example, the zeroth-order response Hamiltonian is just the ground-state KS-Hamiltonian, which depends only on the ground-state density. The first-order response Hamiltonian

$$
\hat{H}_{\mathrm{KS}}^{(1)}\left[n\left(\boldsymbol{r}^{\prime}, t\right)\right](\boldsymbol{r})=\int \mathrm{d}^{3} r^{\prime} f_{\mathrm{Hxc}}\left[n^{(0)}\right]\left(\boldsymbol{r}, \boldsymbol{r}^{\prime}\right) n^{(1)}\left(\boldsymbol{r}^{\prime}, t\right)
$$

has the first-order Hartree-exchange-correlation kernel $f_{\mathrm{Hxc}}\left[n^{(0)}\right]\left(\boldsymbol{r}, \boldsymbol{r}^{\prime}\right)$, which depends on the ground-state density $n^{(0)}(\boldsymbol{r})$, multiplied by the first-order density response $n^{(1)}(\boldsymbol{r}, t)$.

Now, by equating different orders of $\lambda$ in the TD-KS equation, we obtain in zeroth order

$$
\mathrm{i} \frac{\partial}{\partial t} \varphi_{k}^{(0)}(\boldsymbol{r}, t)=\hat{H}_{\mathrm{KS}}^{(0)}\left[n^{(0)}\right](\boldsymbol{r}) \varphi_{k}^{(0)}(\boldsymbol{r}, t),
$$

in first order,

$$
\begin{aligned}
\mathrm{i} \frac{\partial}{\partial t} \varphi_{k}^{(1)}(\boldsymbol{r}, t)=\hat{H}_{\mathrm{KS}}^{(0)}\left[n^{(0)}\right](\boldsymbol{r}) & \varphi_{k}^{(1)}(\boldsymbol{r}, t) \\
& +\left[\hat{H}_{\mathrm{KS}}^{(1)}[n](\boldsymbol{r}, t)+v_{\mathrm{ext}}^{(1)}(\boldsymbol{r}, t)\right] \varphi_{k}^{(0)}(\boldsymbol{r}, t),
\end{aligned}
$$

\footnotetext{
${ }^{5}$ Remember that we are working within the adiabatic approximation here, and therefore, the TD-KS Hamiltonian has no memory.
} 
in second order,

$$
\begin{aligned}
\mathrm{i} \frac{\partial}{\partial t} \varphi_{k}^{(2)}(\boldsymbol{r}, t)=\hat{H}_{\mathrm{KS}}^{(0)}\left[n^{(0)}\right](\boldsymbol{r}) \varphi_{k}^{(2)}(\boldsymbol{r}, t) \\
+\left(\hat{H}_{\mathrm{KS}}^{(1)}[n](\boldsymbol{r}, t)+v_{\mathrm{ext}}^{(1)}(\boldsymbol{r}, t)\right) \varphi_{k}^{(1)}(\boldsymbol{r}, t) \\
\quad+\left(\hat{H}_{\mathrm{KS}}^{(2)}[n](\boldsymbol{r}, t)+v_{\mathrm{ext}}^{(2)}(\boldsymbol{r}, t)\right) \varphi_{k}^{(0)}(\boldsymbol{r}, t),
\end{aligned}
$$

and so on. The equations form a hierarchy, where higher-order responses can be calculated from the lower-order ones [Gonze 1989, Gonze 1995b].

The above equations still depend on time in a non-trivial way (except the zeroth order which is just the trivial time-propagation of the ground-state). Nevertheless, the only explicit time-dependence is in the time-dependent external potential. If the potential has only one frequency, the linear response will also have only one frequency. If the potential has two frequencies, the linear response has two. However, the second-order response will have frequencies which are sums and differences of the original frequencies. For example, in case of one frequency $\omega$, the second-order response has frequency $\omega-\omega=0$ and frequency $\omega+\omega=2 \omega$. Furthermore, in the case of two frequencies $\omega_{1}$ and $\omega_{2}$, the second-order response has frequencies $0,2 \omega_{1}, 2 \omega_{2}$, $\omega_{1}+\omega_{2}$, and $\left|\omega_{1}-\omega_{2}\right|$. The 3rd-order mixes three frequencies, and in addition to the frequencies of the field, it can also mix the frequencies generated by the 2nd-order response. Each new order brings new mixed frequencies.

From this point onward, we assume that we have only a single frequency $\omega$ in the external field. The external field reads

$$
v_{\text {ext }}^{(1)}(\boldsymbol{r}, t)=v_{\text {ext }}^{+\omega}(\boldsymbol{r}) \mathrm{e}^{+i \omega t}+v_{\text {ext }}^{-\omega}(\boldsymbol{r}) \mathrm{e}^{-i \omega t},
$$

or, if we choose to use a cosine field,

$$
v_{\text {ext }}^{(1)}(\boldsymbol{r}, t)=\frac{1}{2} v_{\text {ext }}^{\omega}(\boldsymbol{r}) \mathrm{e}^{+i \omega t}+\frac{1}{2} v_{\text {ext }}^{\omega}(\boldsymbol{r}) \mathrm{e}^{-i \omega t}=v_{\text {ext }}^{\omega}(\boldsymbol{r}) \cos \omega t .
$$

A general first-order wavefunction in this case can be written as

$$
\begin{aligned}
\varphi(\boldsymbol{r}, t)=\mathrm{e}^{-\mathrm{i} \varepsilon^{(0)} t-\mathrm{i} \lambda \Delta \varepsilon^{(1)}(t)} \\
\times\left\{\varphi^{(0)}(\boldsymbol{r})+\lambda\left[\varphi_{+\omega}^{(1)}(\boldsymbol{r}) \mathrm{e}^{\mathrm{i} \omega t}+\varphi_{-\omega}^{(1)}(\boldsymbol{r}) \mathrm{e}^{-\mathrm{i} \omega t}\right]\right\}+\mathcal{O}\left(\lambda^{2}\right),
\end{aligned}
$$

where $\varphi_{\omega}(\boldsymbol{r})$ are now time-independent, and we have included a time-dependent level shift

$$
\Delta \varepsilon^{(1)}[n](t)=\int_{-\infty}^{t} \mathrm{~d} t\left\langle\varphi^{(0)}\left|\hat{H}_{\mathrm{KS}}^{(1)}[n](t)+v_{\mathrm{ext}}^{(1)}(t)\right| \varphi^{(0)}\right\rangle .
$$

The first-order level shift $\Delta \varepsilon^{(1)}(t)$ is a first order correction to the phase of the zeroth-order wavefunction caused by the first-order Hamiltonian. By 
including it, we keep the first-order wavefunction $\varphi_{ \pm \omega}^{(1)}$ orthogonal to the zeroth-order wavefunction $\varphi^{(0)}$. Otherwise, $\varphi_{ \pm \omega}^{(1)}$ would be time-dependent and include a time-dependent $\varphi^{(0)}$ component in order to correct the phase [Langhoff 1972].

From the wavefunction, we obtain the response densities. The zeroth-order density is just the ground-state density

$$
n^{(0)}(\boldsymbol{r}, t)=\sum_{k} n_{k}\left|\varphi_{k}^{(0)}(\boldsymbol{r})\right|^{2}
$$

and the first-order response density

$$
\begin{aligned}
n^{(1)}(\boldsymbol{r}, t)= & \sum_{k} n_{k}\left\{\left[\varphi_{k}^{(0)}(\boldsymbol{r})\right]^{*} \varphi_{k,+\omega}^{(1)}(\boldsymbol{r}) \mathrm{e}^{\mathrm{i} \omega t}+\left[\varphi_{k}^{(0)}(\boldsymbol{r})\right]^{*} \varphi_{k,-\omega}^{(1)}(\boldsymbol{r}) \mathrm{e}^{-\mathrm{i} \omega t}\right. \\
& \left.+\left[\varphi_{k,+\omega}^{(1)}(\boldsymbol{r})\right]^{*} \varphi_{k}^{(0)}(\boldsymbol{r}) \mathrm{e}^{-\mathrm{i} \omega t}+\left[\varphi_{k,-\omega}^{(1)}(\boldsymbol{r})\right]^{*} \varphi_{k}^{(0)}(\boldsymbol{r}) \mathrm{e}^{\mathrm{i} \omega t}\right\} \\
= & \sum_{k} n_{k}\left\{\left[\varphi_{k}^{(0)}(\boldsymbol{r})\right]^{*} \varphi_{k,+\omega}^{(1)}(\boldsymbol{r})+\left[\varphi_{k,-\omega}^{(1)}(\boldsymbol{r})\right]^{*} \varphi_{k}^{(0)}(\boldsymbol{r})\right\} \mathrm{e}^{\mathrm{i} \omega t}+\mathrm{cc}
\end{aligned}
$$

is oscillating at the frequency $\omega$ as expected.

Next, we insert the guess wavefunction to the TDKS equation and expand it up to first order. On the left-hand-side, we obtain

$$
\begin{aligned}
i \frac{\partial}{\partial t}\left(\varphi_{k}^{(0)}(\boldsymbol{r})\right. & \left.+\lambda \varphi_{k,+\omega}^{(1)}(\boldsymbol{r}) \mathrm{e}^{\mathrm{i} \omega t}+\lambda \varphi_{k,-\omega}^{(1)}(\boldsymbol{r}) \mathrm{e}^{-\mathrm{i} \omega t}\right) \mathrm{e}^{-\mathrm{i} \varepsilon_{k}^{(0)} t-\mathrm{i} \lambda \Delta \varepsilon_{k}^{(1)}(t)} \\
= & \mathrm{e}^{-\mathrm{i} \varepsilon_{k}^{(0)} t-\mathrm{i} \lambda \Delta \varepsilon_{k}^{(1)}(t)}\left\{\left[\varepsilon_{k}^{(0)}+\lambda \frac{\partial}{\partial t} \Delta \varepsilon_{k}^{(1)}(t)\right] \varphi_{k}^{(0)}(\boldsymbol{r})\right. \\
+\lambda(-\omega & \left.\left.+\varepsilon_{k}^{(0)}\right) \varphi_{k,+\omega}^{(1)}(\boldsymbol{r}) \mathrm{e}^{\mathrm{i} \omega t}+\lambda\left(\omega+\varepsilon_{k}^{(0)}\right) \varphi_{k,-\omega}^{(1)}(\boldsymbol{r}) \mathrm{e}^{-\mathrm{i} \omega t}\right\}+\mathcal{O}\left(\lambda^{2}\right) .
\end{aligned}
$$

On the right-hand-side, we obtain

$$
\begin{gathered}
\left\{\hat{H}_{\mathrm{KS}}^{(0)}\left[n^{(0)}\right](\boldsymbol{r}) \varphi_{k}^{(0)}(\boldsymbol{r})+\lambda \hat{H}_{\mathrm{KS}}^{(0)}\left[n^{(0)}\right](\boldsymbol{r})\left[\varphi_{k,+\omega}^{(1)}(\boldsymbol{r}) \mathrm{e}^{\mathrm{i} \omega t}+\varphi_{k,-\omega}^{(1)}(\boldsymbol{r}) \mathrm{e}^{-\mathrm{i} \omega t}\right]\right. \\
\left.+\lambda\left[\int \mathrm{d}^{3} r^{\prime} f_{\mathrm{Hxc}}\left[n^{(0)}\right]\left(\boldsymbol{r}, \boldsymbol{r}^{\prime}\right) n^{(1)}\left(\boldsymbol{r}^{\prime}, t\right)+v_{\mathrm{ext}}^{(1)}(\boldsymbol{r}, t)\right] \varphi_{k}^{(0)}(\boldsymbol{r})\right\} \\
\times \mathrm{e}^{-\mathrm{i} \varepsilon_{k}^{(0)} t-\mathrm{i} \lambda \Delta \varepsilon_{k}^{(1)}(t)}+\mathcal{O}\left(\lambda^{2}\right) .
\end{gathered}
$$

The first-order equation can now be written in matrix form by gathering terms proportional to the resonant part $\mathrm{e}^{\mathrm{i} \omega t-\mathrm{i} \varepsilon_{k}^{(0)} t-\mathrm{i} \lambda \Delta \varepsilon_{k}^{(1)}(t)}$ and to the anti- 
resonant part $\mathrm{e}^{-\mathrm{i} \omega t-\mathrm{i} \varepsilon_{k}^{(0)} t-\mathrm{i} \lambda \Delta \varepsilon_{k}^{(1)}(t)}$ :

$$
\begin{aligned}
\left(\begin{array}{cc}
\hat{H}_{\mathrm{KS}}^{(0)}-\varepsilon_{k}^{(0)}+\omega & 0 \\
0 & \hat{H}_{\mathrm{KS}}^{(0)}-\varepsilon_{k}^{(0)}-\omega
\end{array}\right)\left(\begin{array}{c}
\varphi_{k,+\omega}^{(1)} \\
\varphi_{k,-\omega}^{(1)}
\end{array}\right) \\
=-\left(\begin{array}{l}
\left(\begin{array}{l}
v_{\mathrm{Hxc},+\omega}^{(1)}+v_{\mathrm{ext},+\omega}^{(1)}-\varepsilon_{k,+\omega}^{(1)} \\
(1)
\end{array}\left(\begin{array}{l}
(1) \\
v_{\mathrm{Hxc},-\omega}^{(0)}+v_{\mathrm{ext},-\omega}^{(1)}-\varepsilon_{k,-\omega}^{(1)}
\end{array}\right)\right. \\
\varphi_{k}^{(0)}
\end{array}\right)
\end{aligned}
$$

where

$$
\begin{gathered}
v_{\mathrm{Hxc}, \pm \omega}^{(1)} \mathrm{e}^{ \pm \mathrm{i} \omega t}=\int \mathrm{d}^{3} r^{\prime} f_{\mathrm{Hxc}}\left[n^{(0)}\right]\left(\boldsymbol{r}, \boldsymbol{r}^{\prime}\right) n_{ \pm \omega}^{(1)}\left(\boldsymbol{r}^{\prime}, t\right) \\
n_{ \pm \omega}^{(1)}(\boldsymbol{r}, t)=\sum_{k} n_{k}\left\{\left[\varphi_{k}^{(0)}(\boldsymbol{r})\right]^{*} \varphi_{k, \pm \omega}^{(1)}(\boldsymbol{r})+\left[\varphi_{k, \mp \omega}^{(1)}(\boldsymbol{r})\right]^{*} \varphi_{k}^{(0)}(\boldsymbol{r})\right\} \mathrm{e}^{ \pm \mathrm{i} \omega t}
\end{gathered}
$$

and $\varepsilon_{k, \pm \omega}^{(1)}$ is the Fourier transform of $\frac{\partial}{\partial t} \Delta \varepsilon_{k}^{(1)}(t)$ :

$$
\varepsilon_{k, \pm \omega}^{(1)}=\left\langle\varphi_{k}^{(0)}\left|v_{\mathrm{Hxc}, \pm \omega}^{(1)}+v_{\mathrm{ext}, \pm \omega}^{(1)}\right| \varphi_{k}^{(0)}\right\rangle
$$

In this form, the Sternheimer method looks like a set of linear equations, but in reality it is a nonlinear set of equations as the right-hand side depends on the solution through $v_{\mathrm{Hxc}, \pm \omega}^{(1)}$ which depends on $n_{ \pm \omega}^{(1)}$ and therefore on $\varphi_{k, \pm \omega}^{(1)}$. The usual way is to introduce a self-consistent field (SCF) iteration for the response density $n_{ \pm \omega}^{(1)}$, as for the ground-state DFT problem. This is the essence of the Baroni-Gianozzi-Testa (BGT) method [Baroni 1987b], originally used for static perturbations but equally applicable to TDDFT [Andrade 2007]. A related Lanczos superoperator scheme for efficient calculation of complete spectra is also available [Walker 2006], which is discussed in .

By projecting the Sternheimer equation onto the unperturbed wavefunctions, one obtains the sum-over-states expression in second-order perturbation theory for the wavefunction:

$$
\varphi_{k, \omega}^{(1)}=\sum_{m \neq k}\left|\varphi_{m}^{(0)}\right\rangle \frac{\left\langle\varphi_{m}^{(0)}\left|\hat{H}_{\omega}^{(1)}\right| \varphi_{k}^{(0)}\right\rangle}{\varepsilon_{m}^{(0)}-\varepsilon_{k}^{(0)}+\omega}
$$

where $\hat{H}_{\omega}^{(1)}=v_{\mathrm{Hxc}, \omega}^{(1)}+v_{\mathrm{ext}, \omega}^{(1)}$. Using the Sternheimer equation has the great advantage that it avoids the need for explicit calculation of the unoccupied states that would occur in this sum over states.

As with the time-propagation approach, we have to include an artificial lifetime. Otherwise, i) the matrix is singular when $\omega$ corresponds to the KSeigenvalue difference $\varepsilon_{a}-\varepsilon_{i}$ (an excitation in the independent particle picture), or ii) the response becomes infinite when $\omega$ corresponds to a resonance 
(an excitation in the interacting picture). The former is simply a numerical issue, but the later one has physical meaning and will be used to derive Casida's equation in the next subsection. The artificial lifetime is introduced by multiplying the first-order wavefunction $\varphi_{k}^{(1)}(\boldsymbol{r}, t)$ and the external potential $v_{\text {ext }}(\boldsymbol{r}, t)$ by a decay term $\mathrm{e}^{-\eta t}$. In the first order, the matrix equation then reads

$$
\begin{array}{r}
\left(\begin{array}{cc}
\hat{H}_{\mathrm{KS}}^{(0)}-\varepsilon_{k}^{(0)}+\omega+\mathrm{i} \eta & 0 \\
0 & \hat{H}_{\mathrm{KS}}^{(0)}-\varepsilon_{k}^{(0)}-\omega+\mathrm{i} \eta
\end{array}\right)\left(\begin{array}{c}
\varphi_{k,+\omega}^{(1)} \\
\varphi_{k,-\omega}^{(1)}
\end{array}\right) \\
=-P_{\mathrm{c}}\left(\begin{array}{l}
\left(\begin{array}{c}
v_{\mathrm{Hxc},+\omega}^{(1)}+v_{\mathrm{ext},+\omega}^{(1)} \\
(1) \\
\left.v_{\mathrm{Hxc},-\omega}^{(1)}+v_{\mathrm{ext},-\omega}^{(1)}\right)
\end{array}\right) \varphi_{k}^{(0)} \\
\varphi_{k}^{(0)}
\end{array}\right)
\end{array}
$$

The matrix is no longer singular, and the resonances become Lorentzians with width $\eta$ instead of delta functions. We also added a projector to the unoccupied space $P_{\mathrm{c}}=1-P_{\mathrm{occ}}$, which orthogonalizes the KS response wavefunctions with respect to the occupied KS ground-state wavefunctions. The components of the response wavefunctions in the occupied subspace are not needed because they cancel out in the density response. The projector avoids solving for these (possibly large) components, making the numerical solution more efficient and stable [Baroni 2001, Andrade 2007]. It also simplifies the equation by removing the level shift $\Delta \varepsilon_{ \pm \omega}^{(1)}$. Finally, after the self-consistent solution is found, the linear density response is directly available from equation (1.45).

The Sternheimer equation is particularly suited to the calculation of higher-order response, because solution of only the first-order problem can actually give access to up to third-order derivatives of the total energy (secondorder response), as stated by the $2 n+1$ Theorem for DFT [Gonze 1989]. Variational principles can be used to show that $\left\{\phi_{i}^{(n)}\right\}$, the derivatives of order $n$ of the KS wavefunctions with respect to a perturbation, can be used to construct all derivatives of the total energy up to order $2 n+1$. Consider a bare external perturbation $\hat{H}_{\text {bare }}^{(n)}$ and a total perturbation $\hat{H}^{(n)}$, including Hartree and exchange-correlation response (the "local fields" [Hybertsen 1987]). For $n=0$, this theorem reduces to the familiar Hellman-Feynman Theorem, used in calculation of forces from only ground-state quantities:

$$
E^{(1)}=\frac{\partial E}{\partial \lambda}=\sum_{i}^{\text {occ }}\left\langle\varphi_{i}\left|\hat{H}_{\text {bare }}^{(1)}\right| \varphi_{i}\right\rangle
$$

At $n=1$, the expression for the second derivative (first-order response) is

$$
E^{(2)}=\sum_{i}^{\text {occ }}\left[\left\langle\varphi_{i}^{(0)}\left|\hat{H}^{(1)}\right| \varphi_{i}^{(1)}\right\rangle+\text { cc. }+\left\langle\varphi_{i}^{(0)}\left|\hat{H}_{\text {bare }}^{(2)}\right| \varphi_{i}^{(0)}\right\rangle\right]
$$


and for the third derivative (second-order response) are

$$
\begin{aligned}
& E^{(3)}=\sum_{i}^{\text {occ }}\left[\left\langle\varphi_{i}^{(1)}\left|\hat{H}^{(1)}\right| \varphi_{i}^{(1)}\right\rangle+\left\langle\varphi_{i}^{(1)} \mid \varphi_{i}^{(1)}\right\rangle\left\langle\varphi_{i}^{(0)}\left|\hat{H}^{(1)}\right| \varphi_{i}^{(0)}\right\rangle\right. \\
& \left.+\left\langle\varphi_{i}^{(1)}\left|\hat{H}_{\text {bare }}^{(2)}\right| \varphi_{i}^{(0)}\right\rangle+\text { cc. }+\left\langle\varphi_{i}^{(0)}\left|\hat{H}_{\text {bare }}^{(3)}\right| \varphi_{i}^{(0)}\right\rangle\right] \\
& +\frac{1}{6} \int \frac{\delta^{3} E\left[n^{(0)}\right]}{\delta n(\boldsymbol{r}) \delta n\left(\boldsymbol{r}^{\prime}\right) \delta n\left(\boldsymbol{r}^{\prime \prime}\right)} n^{(1)}(\boldsymbol{r}) n^{(1)}\left(\boldsymbol{r}^{\prime}\right) n^{(1)}\left(\boldsymbol{r}^{\prime \prime}\right) d r d r^{\prime} d r^{\prime \prime}
\end{aligned}
$$

where superscripts indicate the order of derivatives with respect to the perturbation [Gonze 1989]. The bare perturbation $\hat{H}_{\text {bare }}^{(n)}$ is zero for $n>1$ for electric fields, but not in general. The third functional derivative here is the second-order kernel $M_{\mathrm{xc}}$.

Conveniently, it turns out even in these equations, only the projection of the wavefunction derivatives onto the unoccupied subspace $P_{c} \varphi_{i}^{(1)}$ are required for this formula [Debernardi 1994], making the Sternheimer calculation more efficient. With this formula for $E^{(3)}$, the Sternheimer approach allows efficient access to phonon anharmonicities and nonlinear optical susceptibilities from solution of the first-order Sternheimer equation. This is true even for mixed derivatives with respect to perturbations in different directions or even entirely different perturbations. To get even higher orders, one can use the hierarchy of Sternheimer equations to solve for $\left\{\varphi_{i}^{(n)}\right\}$ from results at lower orders [Gonze 1989, Gonze 1995b]. The situation becomes somewhat more complicated beyond first order: the entire wavefunctions, not just projections to the unoccupied subspace, are needed, and the issues of phases and normalization are more subtle than in the first-order equation [Strubbe 2011a]. The $2 n+1$ formulae for the energy derivatives at higher orders are straightforward but increasingly lengthy. For the time-dependent case, rather than total energies we use instead the action, or in the frequency domain, the Floquet quasi-energy [Langhoff 1972].

The $2 n+1$ theorem actually also provides an alternate calculation approach for $\varphi^{(1)}$ [Gonze 1995b, Dal Corso 1996, Gonze 1997a, Baroni 2001]. The formula for $E^{(3)}$ is variational with respect to $\varphi^{(1)}$, just as $E$ is variational with respect to $\varphi^{(0)}$, as stated in the standard Variational Theorem of quantum mechanics. As a result, one can solve for $\varphi^{(1)}$ by direct minimization of the functional $E^{(3)}\left[\left\{\varphi_{i}^{(1)}\right\}\right]$. This approach is formally equivalent to solution by self-consistency, and the choice of technique is a question of numerical strategy.

\subsubsection{Casida method}

From the Sternheimer method, we can continue to Casida's equation by writing the linear Sternheimer equation in the particle-hole basis, i.e., in the 
Kohn-Sham orbital basis including unoccupied states. First, we write the first-order response wavefunction as a linear combination of Kohn-Sham orbitals (i.e., sum-over-states expansion)

$$
\varphi_{k}^{(1)}(\boldsymbol{r})=\sum_{a} c_{a}^{(k)} \varphi_{a}^{(0)}(\boldsymbol{r}),
$$

where $c_{a}^{(k)}$ is the coefficient for an electronic excitation from the $k$ th occupied state to the $a$ th basis function $\varphi_{a}^{(0)}$, i.e., some unoccupied KS state. As in Eq. (1.48), we are considering only the projection of $\varphi_{k}^{(1)}(\boldsymbol{r})$ into the unoccupied subspace, which will remove the level shift. We insert this linear combination into the first-order equation and multiply it from the left by $\left\langle\varphi_{b}^{(0)}\right|$, i.e., take an inner product with another basis function. The first-order TD-KS equation (1.43) now reads

$$
\begin{gathered}
\sum_{a}\left(-\omega+\varepsilon_{k}^{(0)}\right) \delta_{a, b} \mathrm{e}^{\mathrm{i} \omega t-\mathrm{i} \varepsilon_{k}^{(0)} t} c_{a}^{(k,+\omega)}+\left(\omega+\varepsilon_{k}^{(0)}\right) \delta_{a, b} \mathrm{e}^{-\mathrm{i} \omega t-\mathrm{i} \varepsilon_{k}^{(0)} t} c_{a}^{(k,-\omega)} \\
=\sum_{a} \varepsilon_{a}^{(0)} \delta_{a, b} \mathrm{e}^{\mathrm{i} \omega t-\mathrm{i} \varepsilon_{k}^{(0)} t} c_{a}^{(k,+\omega)}+\sum_{a} \varepsilon_{a}^{(0)} \delta_{a, b} \mathrm{e}^{\mathrm{i} \omega t-\mathrm{i} \varepsilon_{k}^{(0)} t} c_{a}^{(k,-\omega)} \\
\left.+\left\langle\varphi_{b}^{(0)}\right| f_{\mathrm{Hxc}}\left[n^{(0)}\right]\right) n^{(1)}(t)\left|\varphi_{k}^{(0)}\right\rangle \mathrm{e}^{-\mathrm{i} \varepsilon_{k}^{(0)} t} \\
+\left\langle\varphi_{b}^{(0)}\left|v_{\mathrm{ext}}(t)\right| \varphi_{k}^{(0)}\right\rangle \mathrm{e}^{-\mathrm{i} \varepsilon_{k}^{(0)} t}
\end{gathered}
$$

Writing the equations in a basis allows us to remove the nonlinearity caused by the Hartree-exchange-correlation kernel:

$$
\begin{aligned}
& \left\langle\varphi_{b}^{(0)}\left|\hat{f}_{\mathrm{Hxc}}\left[n^{(0)}\right] n^{(1)}(t)\right| \varphi_{k}^{(0)}\right\rangle \mathrm{e}^{-i \varepsilon_{k}^{(0)} t}=\int \mathrm{d}^{3} r \int \mathrm{d}^{3} r^{\prime} f_{\mathrm{Hxc}}\left[n^{(0)}\right]\left(\boldsymbol{r}, \boldsymbol{r}^{\prime}\right) \\
& \times \sum_{k^{\prime}} n_{k^{\prime}}\left\{\left[\left[\varphi_{k^{\prime}}^{(0)}\left(\boldsymbol{r}^{\prime}\right)\right]^{*} \varphi_{k^{\prime},+\omega}^{(1)}\left(\boldsymbol{r}^{\prime}\right)+\left[\varphi_{k^{\prime},-\omega}^{(1)}\left(\boldsymbol{r}^{\prime}\right)\right]^{*} \varphi_{k^{\prime}}^{(0)}\left(\boldsymbol{r}^{\prime}\right)\right] \mathrm{e}^{\mathrm{i} \omega t}\right. \\
& \left.+\left[\left[\varphi_{k^{\prime}}^{(0)}\left(\boldsymbol{r}^{\prime}\right)\right]^{*} \varphi_{k^{\prime},-\omega}^{(1)}\left(\boldsymbol{r}^{\prime}\right)+\left[\varphi_{k^{\prime},+\omega}^{(1)}\left(\boldsymbol{r}^{\prime}\right)\right]^{*} \varphi_{k^{\prime}}^{(0)}\left(\boldsymbol{r}^{\prime}\right)\right] \mathrm{e}^{-\mathrm{i} \omega t}\right\} \\
& \times \varphi_{b}^{(0)}(\boldsymbol{r}) \varphi_{k}^{(0)}(\boldsymbol{r}) d^{3} r d^{3} r^{\prime}
\end{aligned}
$$

Inserting the expansion for $\varphi^{(1)}$ in terms of the unoccupied KS eigenfunctions gives

$$
\begin{gathered}
\left\langle\varphi_{b}^{(0)}\left|f_{\mathrm{Hxc}}\left[n^{(0)}\right] n^{(1)}(t)\right| \varphi_{k}^{(0)}\right\rangle \mathrm{e}^{-i \varepsilon_{k}^{(0)} t}=\int \mathrm{d}^{3} r \int \mathrm{d}^{3} r^{\prime} f_{\mathrm{Hxc}}\left[n^{(0)}\right]\left(\boldsymbol{r}, \boldsymbol{r}^{\prime}\right) \\
\times \sum_{k^{\prime}, b^{\prime}} n_{k^{\prime}}\left\{\left[\varphi_{k^{\prime}}^{(0)}\left(\boldsymbol{r}^{\prime}\right) c_{b^{\prime}}^{\left(k^{\prime},+\omega\right)} \varphi_{b^{\prime}}^{(0)}\left(\boldsymbol{r}^{\prime}\right)+\left(c_{b^{\prime}}^{\left(k^{\prime},-\omega\right)}\right)^{*} \varphi_{b^{\prime}}^{(0)}\left(\boldsymbol{r}^{\prime}\right) \varphi_{k^{\prime}}^{(0)}\left(\boldsymbol{r}^{\prime}\right)\right] \mathrm{e}^{\mathrm{i} \omega t}\right. \\
\left.+\left[\varphi_{k^{\prime}}^{(0)}\left(\boldsymbol{r}^{\prime}\right) c_{b^{\prime}}^{\left(k^{\prime},-\omega\right)} \varphi_{b^{\prime}}^{(0)}\left(\boldsymbol{r}^{\prime}\right)+\left(c_{b^{\prime}}^{\left(k^{\prime},+\omega\right)}\right)^{*} \varphi_{b^{\prime}}^{(0)}\left(\boldsymbol{r}^{\prime}\right) \varphi_{k^{\prime}}^{(0)}\left(\boldsymbol{r}^{\prime}\right)\right] \mathrm{e}^{-\mathrm{i} \omega t}\right\} \\
\times \varphi_{b}^{(0)}(\boldsymbol{r}) \varphi_{k}^{(0)}(\boldsymbol{r}) d^{3} r d^{3} r^{\prime}
\end{gathered}
$$


This finally leads to

$$
\begin{aligned}
& \left\langle\varphi_{b}^{(0)}\left|f_{\mathrm{Hxc}}\left[n^{(0)}\right] n^{(1)}(t)\right| \varphi_{k}^{(0)}\right\rangle \mathrm{e}^{-i \varepsilon_{k}^{(0)} t}= \\
& \sum_{k^{\prime}, b^{\prime}} n_{k^{\prime}} K_{b k, b^{\prime} k^{\prime}}\left\{\left[c_{b^{\prime}}^{\left(k^{\prime},+\omega\right)}+\left(c_{b^{\prime}}^{\left(k^{\prime},-\omega\right)}\right)^{*}\right] \mathrm{e}^{\mathrm{i} \omega t}+\left[c_{b^{\prime}}^{\left(k^{\prime},-\omega\right)}+\left(c_{b^{\prime}}^{\left(k^{\prime},+\omega\right)}\right)^{*}\right] \mathrm{e}^{-\mathrm{i} \omega t}\right\},
\end{aligned}
$$

where

$$
K_{b k, b^{\prime} k^{\prime}}=\int \mathrm{d}^{3} r \int \mathrm{d}^{3} r^{\prime} f_{\mathrm{Hxc}}\left[n^{(0)}\right]\left(\boldsymbol{r}, \boldsymbol{r}^{\prime}\right) \varphi_{k^{\prime}}^{(0)}\left(\boldsymbol{r}^{\prime}\right) \varphi_{b^{\prime}}^{(0)}\left(\boldsymbol{r}^{\prime}\right) \varphi_{b}^{(0)}(\boldsymbol{r}) \varphi_{k}^{(0)}(\boldsymbol{r})
$$

is the Hartree-exchange-correlation matrix element for interaction of excitations $b \leftarrow k$ and $b^{\prime} \leftarrow k^{\prime}$ ( $k$ and $k^{\prime}$ are occupied orbitals, $b$ and $b^{\prime}$ unoccupied). This term couples independent-particle excitations (KS-eigenvalue differences) to interacting-particle excitations (TD-KS transition frequencies). Remember that we assumed the KS orbitals to be real functions.

The matrix form of the Sternheimer equation in the particle-hole basis reads as

$$
\begin{gathered}
\left(\begin{array}{cccc}
\Delta E+\omega I & 0 & -\eta & 0 \\
0 & \Delta E-\omega I & 0 & -\eta \\
\eta & 0 & \Delta E+\omega I & 0 \\
0 & \eta & 0 & \Delta E-\omega I
\end{array}\right)\left(\begin{array}{l}
C^{(+\omega, r e)} \\
C^{(-\omega, r e)} \\
C^{(+\omega, i m)} \\
C^{(-\omega, i m)}
\end{array}\right) \\
=-\left(\begin{array}{cccc}
K & K & 0 & 0 \\
K & K & 0 & 0 \\
0 & 0 & K & -K \\
0 & 0 & -K & K
\end{array}\right) N\left(\begin{array}{l}
C^{(+\omega, r e)} \\
C^{(-\omega, r e)} \\
C^{(+\omega, i m)} \\
C^{(-\omega, i m)}
\end{array}\right)-N^{1 / 2}\left(\begin{array}{l}
V_{+\omega, r e} \\
V_{-\omega, r e} \\
V_{+\omega, i m} \\
V_{-\omega, i m}
\end{array}\right)
\end{gathered}
$$

where real and imaginary parts have been separated, $\Delta E_{b k, b^{\prime} k^{\prime}}=\delta_{k, k^{\prime}} \delta_{b^{\prime}, b^{\prime}}\left(\varepsilon_{b}-\right.$ $\left.\varepsilon_{k}\right), N_{b k, b^{\prime} k^{\prime}}=\delta_{k, k^{\prime}} \delta_{b, b^{\prime}} n_{k^{\prime}}, V_{b k}^{ \pm \omega}=\left\langle\varphi_{b}^{(0)}\left|v_{\text {ext }, \pm \omega}\right| \varphi_{k}^{(0)}\right\rangle$ and $K$ is the above Hartree-exchange-correlation kernel matrix. As one can easily see, the nonlinearity is has been eliminated, i.e., the above equation is a linear equation if the first term on the right-hand side is moved to the left:

$$
\begin{aligned}
& {\left[\left(\begin{array}{cccc}
-\Delta E-\mathcal{K} & -\mathcal{K} & \eta & 0 \\
\mathcal{K} & \Delta E+\mathcal{K} & 0 & -\eta \\
-\eta & 0 & -\Delta E-\mathcal{K} & \mathcal{K} \\
0 & \eta & -\mathcal{K} & \Delta E+\mathcal{K}
\end{array}\right)-\omega I\right]} \\
& \times\left(\begin{array}{c}
B^{(+\omega, r e)} \\
B^{(-\omega, r e)} \\
B^{(+\omega, i m)} \\
B^{(-\omega, i m)}
\end{array}\right)=-N\left(\begin{array}{c}
-V_{+\omega, r e} \\
V_{-\omega, r e} \\
-V_{+\omega, i m} \\
V_{-\omega, i m}
\end{array}\right),
\end{aligned}
$$


where $\mathcal{K}=N^{1 / 2} K N^{1 / 2}, B=N^{1 / 2} C$, and we have already modified it slightly for convenience in the rest of the discussion.

In the limit when the lifetime parameter $\eta$ goes to zero (i.e., infinite lifetime), the matrix has singularities at certain frequencies. As we included the Hartree-exchange-correlation kernel in the matrix, the response has poles only at the interacting resonance frequencies, and not at the noninteracting resonance frequencies as Eq. (1.48) did. Therefore, we can find the interacting resonance frequencies in the limit $\eta \rightarrow 0$ by finding the singularities of the matrix.

The matrix is a $2 \times 2$ block-diagonal system in the limit $\eta \rightarrow 0$, and the second diagonal block is the transpose of the first one. The blocks have the same eigenvalues, but the right and left eigenvectors of the blocks are swapped. We can focus on the first block and find the eigenvalues of the following equation:

$$
\left[\left(\begin{array}{cc}
-\Delta E-\mathcal{K} & -\mathcal{K} \\
\mathcal{K} & \Delta E+\mathcal{K}
\end{array}\right)-\omega I\right]\left(\begin{array}{c}
-B^{(+\omega, r e)} \\
B^{(-\omega, r e)}
\end{array}\right)=0 .
$$

We apply an unitary transformation $Q=\frac{1}{\sqrt{2}}\left(\begin{array}{cc}1 & 1 \\ -1 & 1\end{array}\right)$, and multiply from left by $\Delta E^{\frac{1}{2}}$ to obtain

$$
\left[\left(\begin{array}{cc}
0 & \Delta E \\
\Delta E+2 \Delta E^{\frac{1}{2}} \mathcal{K} \Delta E^{-\frac{1}{2}} & 0
\end{array}\right)-\omega I\right]\left(\begin{array}{c}
\Delta E^{\frac{1}{2}}\left[B^{(+\omega, r e)}+B^{(-\omega, r e)}\right] \\
\Delta E^{\frac{1}{2}}\left[-B^{(+\omega, r e)}+B^{(-\omega, r e)}\right]
\end{array}\right)=0 .
$$

The determinant of the matrix inside the square brackets can be easily calculated as $\Delta E$ and $\omega I$ are diagonal. Setting the determinant equal to zero gives us the eigenvalue equation

$$
\Delta E^{2}+2 \Delta E^{\frac{1}{2}} \mathcal{K} \Delta E^{\frac{1}{2}}=\omega^{2} I
$$

or, when we expand $\mathcal{K}=N^{\frac{1}{2}} K N^{\frac{1}{2}}$,

$$
\Delta E^{2}+2 \Delta E^{\frac{1}{2}} N^{\frac{1}{2}} K N^{\frac{1}{2}} \Delta E^{\frac{1}{2}}=\omega^{2} I,
$$

which is the well-known Casida's equation with one small difference: instead of differences of occupation numbers $\left(n_{a}-n_{i}\right)$, the actual occupation numbers appear. This is a consequence of our assumption of integral occupations. The extension to fractional occupations will be discussed in the next subsection.

The eigenvectors $F$ of Casida's equation, Eq. (1.63), can be used to extract the strength of the response to the external field. After some algebra, for example, the polarizability can be written as

$$
\alpha_{\gamma \delta}(\omega)=\mu_{\gamma} N^{\frac{1}{2}} \Delta E^{\frac{1}{2}} \sum_{k} F_{k}\left(\omega_{k}^{2}-\omega^{2}\right)^{-1} F_{k}^{\dagger} \Delta E^{\frac{1}{2}} N^{\frac{1}{2}} \mu_{\delta},
$$

where $\mu_{\delta}$ is the dipole-moment operator in direction $\delta$, with matrix elements in the particle-hole basis $\mu_{\delta, a i}=\left\langle\varphi_{a}^{(0)}\left|r_{\delta}\right| \varphi_{i}^{(0)}\right\rangle$. 


\subsubsection{Generalizations and discussion}

In this subsection, we will discuss what changes if we do not make the assumptions of the beginning of the section. We start with the first assumption - the adiabatic approximation. Without the adiabatic approximation, the exchange-correlation functional has memory, i.e., the exchange-correlation functional depends on density at all previous times. In principle, it is trivial for the time-propagation method. We only have to store all previous densities and calculate the exchange-correlation potential from these. In practice, this is very demanding task and often impossible beyond model systems.

In the Sternheimer method, memory will show up as a frequency dependence of the exchange-correlation kernels. At first order, the kernel depends only on one frequency, $f_{\mathrm{xc}}\left[n_{\mathrm{GS}}\right]\left(\boldsymbol{r}, \boldsymbol{r}^{\prime}, \omega\right)$, but at higher orders it depends on multiple frequencies. Again, if explicit forms of the frequency-dependent kernels are known, it is straightforward to include memory in principle. However, a practical implementation might not be easy and it will depend a lot on the actual form of the memory-dependence in the functionals, which remains an important unresolved theoretical issue [see ].

In the case of the Casida method, the matrix becomes frequency-dependent [Casida 1995], which means that the linear eigenvalue problem becomes a nonlinear eigenvalue problem. A nonlinear eigenvalue problem is much harder to solve than a linear eigenvalue problem (e.g., SCF iterations may be required).

If a system is spin-polarized, each spin has its own exchange-correlation potential $v_{\mathrm{xc}}^{\alpha}$ and $v_{\mathrm{xc}}^{\beta}$. The exchange-correlation kernel is replaced by three exchange-correlation kernels $f_{\mathrm{xc}}^{\alpha \alpha}, f_{\mathrm{xc}}^{\beta \beta}$, and $f_{\mathrm{xc}}^{\alpha \beta}$ [Casida 1995, Guan 2000].

If a system has fractional occupation numbers, an excitation which happens from one partially occupied state $i$ to another partially occupied state $j$ will have an opposite excitation (or de-excitation) from $j$ to $i$. The expressions from perturbation theory now contains the occupation difference $n_{i}-n_{j}$. The original presentation of the Casida method [Casida 1995] shows this general case. The situation for the Sternheimer method is significantly more complicated due to the question of what happens to the projection onto the unoccupied subspace. For metals, smearing is generally needed for reasonable convergence of the ground-state problem with respect to $k$-point sampling. A computational scheme has been derived to solve the Sternheimer equation when the occupation function corresponds to a smearing, which is generally needed for reasonable convergence of the ground state of metals with respect to $k$-point sampling [de Gironcoli 1995]. Smearing methods such as Gaussian, Methfessel-Paxton, and "cold smearing" provide an occupation function $\tilde{\theta}(x)$ depending on energy and satisfying certain properties of the Fermi-Dirac thermal distribution. In this case, introduction of some extra projectors into the Sternheimer equation allows the density response

$$
n^{(1)}=\sum_{i j} \frac{\tilde{\theta}\left(\varepsilon_{\mathrm{F}}-\varepsilon_{i}\right)-\tilde{\theta}\left(\varepsilon_{\mathrm{F}}-\varepsilon_{j}\right)}{\varepsilon_{i}-\varepsilon_{j}} \varphi_{i}^{*} \varphi_{j}\left\langle\varphi_{j}\left|\hat{H}_{\mathrm{bare}}^{(1)}\right| \varphi_{i}\right\rangle
$$


to be written in the same form as Eq. (1.45) for the zero-temperature (semiconducting) case in which all states are full or empty. The smearing functions give the correct answer only in the limit of zero smearing width, and are designed to improve convergence while minimizing the error with respect to the unsmeared case; by contrast the Fermi-Dirac distribution can be used to describe physical temperature effects. In both the static and dynamic case, one must take care about what physical ensemble the system is supposed to be in, to determine whether the assumption that the occupations and Fermi level are not affected by the perturbation is appropriate [Baroni 2001]. It is also possible to generalize the smearing scheme to the case of arbitrary occupations, for a distribution that does not satisfy the properties of a smearing function or is not even a function of energy at all [Strubbe 2011b]. Finally, if we use $k$-points, the ground-state KS wavefunctions become complex and we cannot obtain the the Casida's equation (1.63). However, we can still obtain a similar eigenvalue equation [Reining 2002].

\subsection{Applications of linear response}

Having reviewed different methods of obtaining response functions, we will now consider the different perturbations that can be studied and how their response functions relate to physical quantities of interest. Electric, magnetic, structural, and $\boldsymbol{k} \cdot \boldsymbol{p}$ perturbations, as well as mixed perturbations, are commonly used to extract both static and dynamic response properties.

\subsubsection{Response to electric perturbations}

We will begin by considering electric perturbations, because they give access to optical properties and account for the vast majority of applications of TDDFT. In molecules, the basic quantity is the polarizability $\alpha$, defined as the response of the dipole to an electric field $\mathcal{E}$, in the limit of zero applied field:

$$
\alpha_{i j}(\omega)=\frac{\partial \mu_{i}}{\partial \mathcal{E}_{j, \omega}}=-\frac{\partial^{2} E}{\partial \mathcal{E}_{i, \omega} \partial \mathcal{E}_{j, \omega}}
$$

The cross-section for optical absorption (in the dipole approximation) can be calculated from the imaginary part:

$$
\sigma_{i j}(\omega)=\frac{4 \pi \omega}{c} \operatorname{Im} \alpha_{i j}(\omega)
$$

The static polarizability (which is purely real) is commonly calculated by finite differences of applied field [Vila 2010], and the dynamic polarizability can be computed by time-propagation [Yabana 1996], typically via application of an instantaneous pulsed electric field, which contains all frequencies. A Fourier transformation of the resulting free oscillations of the dipole moments yields the polarizability. The absorption spectrum is most often calculated by 
the Casida method [Casida 1995, Jamorski 1996], which was designed for this problem; it calculates excited states, and a specific perturbation only enters in the calculation of oscillator strengths. It can be difficult to converge the real part of the dynamic polarizability below the optical gap in this method [Jamorski 1996], in which case it is more efficient to do the calculation via the Sternheimer equation [Andrade 2007]. The electric field appears as a term $\mathcal{E} \cdot \boldsymbol{r}$ in the Hamiltonian, so the perturbation used is $\partial H / \partial \mathcal{E}=\boldsymbol{r}$. This is the response of the dipole moment to a homogeneous electric field, which couples to the dipole, so these are called dipole-dipole polarizabilities. Similar methodologies can be used for dipole-quadrupole polarizabilities (response to a field gradient) and other multipoles [Bishop 1990].

For solids, typically the susceptibility $\chi$ (polarizability per unit cell) and dielectric function $\epsilon$ are used instead of the polarizability, related via

$$
\epsilon=1+4 \pi \chi=1+4 \pi \frac{\alpha}{\mathcal{V}}
$$

where $\mathcal{V}$ is the volume of the unit cell. The optical absorption is calculated just as for finite systems. There is a significant complication in applying a uniform electric field to a periodic system, because the operator $\boldsymbol{r}$ is not periodic. There are two ways to solve the problem: the original solution is to consider spatially modulated fields $\mathcal{E}(\boldsymbol{q})=\mathcal{E}_{0} \mathrm{e}^{i \boldsymbol{q} \cdot \boldsymbol{r}}$, which are periodic although not necessarily commensurate with the cell periodicity. In this case, one can consider the $\boldsymbol{q} \rightarrow 0$ limit to obtain the response to a homogeneous electric field, which is used in TDDFT calculations in the sum-over-states [Hybertsen 1987, Levine 1989] and response-function approaches, which is commonly used for crystals [Olevano 1999, Sagmeister 2009]. It is important to consider carefully the relation between microscopic/macroscopic and transverse/longitudinal responses in this method. Another solution is to use the quantum theory of polarization [Resta 1994, Vanderbilt 2006] to write the operator as $\boldsymbol{r}=i \frac{\partial}{\partial k}$, which is periodic [Gonze 1997a]. The $k$-point derivatives are obtained by finite differences. An equivalent approach is to calculate the polarization within a basis of Wannier functions [Dal Corso 1994]. In either method, we do not study the response of the dipole moment per unit cell, which is not a well-defined quantity, and instead use the polarization. To apply finite homogeneous electric fields in periodic systems, the electricenthalpy approach can be used, in which a term $-\mu \cdot \mathcal{E}$ is added to the total energy functional to be minimized [Souza 2002].

Armed with the dielectric function or polarizability, one can calculate many interesting properties. Inverting the dielectric matrix yields the loss function $\operatorname{Im} \epsilon^{-1}(\boldsymbol{q}, \omega)$, which describes the slowing of energetic electrons in a solid and is measured in electron energy-loss spectroscopy (EELS) experiments [Onida 2002, Marini 2006]. Van der Waals interaction energies can be calculated too: the Hamaker coefficients in the expansion

$$
\Delta E(R)=-\sum_{n=6}^{\infty} \frac{C_{n}}{R^{n}}
$$

Cross Chap. 3 
can be calculated from the Casimir-Polder relation as an integral over polarizabilities evaluated as a function of imaginary frequency. The dominant $C_{6}$ term for interaction of molecules $\mathrm{A}$ and $\mathrm{B}$ is given by

$$
C_{6}^{A B}=\frac{3}{\pi} \int_{0}^{\infty} d u \alpha^{(A)}(i u) \alpha^{(B)}(i u)
$$

Higher-order terms involve higher-order multipole polarizabilities. These coefficients have been calculated by TDDFT with molecular polarizabilities from time-propagation and Sternheimer methods, and surface susceptibilities from response functions, to study molecule-molecule [Marques 2007] and molecule-surface interactions [Botti 2008].

Dielectric response can also be considered not for uniform fields but rather for point charges, giving $\epsilon\left(\boldsymbol{r}, \boldsymbol{r}^{\prime}, \omega\right)$ from a perturbation $1 /\left|\boldsymbol{r}-\boldsymbol{r}^{\prime}\right|$. Timepropagation has been used to study the spatially resolved plasmon response of liquid water [Tavernelli 2006]. This form of the dielectric function can also be used as input for many-body perturbation theory via the $G W$ approximation [Hedin 1969] and Bethe-Salpeter equation. Typically these calculations use the RPA dielectric function, which is equivalent to using only the Hartree response and setting the kernel $f_{\mathrm{xc}}=0$. However, as pointed out in the first practical implementation of this scheme [Hybertsen 1986], using instead the TDDFT $\epsilon$ is an approximate way of including the vertex $\Gamma$. This methodology has been used for quasiparticle and optical calculations on organic molecules [Tiago 2005]. Recently progress has been made in replacing the expensive sums over states with solution of the time-dependent Sternheimer equation [Giustino 2010], which can be done with RPA or including $f_{\mathrm{xc}}$. Response to a related but more exotic perturbation can also be used to parametrize the $\mathrm{DFT}+U$ method, in which projectors on atomic-like orbitals are used to emulate Coulomb repulsion and correct the energies of localized $d$ - and $f$ orbitals in strongly correlated materials [Anisimov 1991]. Ab initio values for $U$ can be calculated from the screened response to a localized potential $\alpha_{I} P_{I}$, where $P_{I}$ is an atomic-orbital projector, implemented via finite differences [Cococcioni 2005].

Response to electric perturbations can be used to calculate nonlinear susceptibilities, describing nonlinear optical properties such as second-harmonic generation, optical rectification, and electrooptical effects [Shen 1984]. The hyperpolarizability $\beta$ of a molecule and second-order susceptibility $\chi^{(2)}$ of a solid are simply the derivatives with respect to field of $\alpha$ and $\chi$, the next order in the Taylor expansion of the dipole moment:

$$
\mu_{i}(\omega)=\mu_{i 0}+\alpha_{i j}(\omega) \mathcal{E}_{j, \omega}+\frac{1}{2} \beta_{i j k}\left(\omega=\omega_{1}+\omega_{2}\right) \mathcal{E}_{j, \omega_{1}} \mathcal{E}_{k, \omega_{2}}+\ldots
$$

though conventions can differ on what numerical factors may appear in this expansion [Willetts 1992]. With the $2 n+1$ theorem, solution of the Sternheimer equation can be used to calculate molecular hyperpolarizabilities 
[Andrade 2007, Vila 2010] as well as the nonlinear susceptibilites of semiconductors with the quantum theory of polarization [Dal Corso 1996]. We are implementing the time-dependent second-order Sternheimer equation for use in nonlinear optics [Strubbe 2011a], in which simultaneous $\boldsymbol{k} \cdot \boldsymbol{p}$ and electric perturbations can be used for the second-order susceptibility in liquids, and two electric perturbations give the higher-order hyperpolarizabilities $\gamma$ and $\delta$ which describe processes such as two-photon absorption and four-wave mixing.

Finite differences are often also used to calculate static hyperpolarizabilities [Vila 2010], and time-propagation can be used for dynamic hyperpolarizabilities; however, the advantage of being able to obtain the entire spectrum from a single calculation is lost, and separate calculations must be done for each set of input frequencies [Takimoto 2007]. The response-function technique has also recently been developed for $\chi^{(2)}$ in the $\boldsymbol{q} \rightarrow 0$ limit, and applied to second-harmonic generation in zincblende semiconductors [Hübener 2010].

\subsubsection{Response to magnetic perturbations}

Magnetic response offers a significant additional challenge compared to electric response because of the fact that the vector potential has to be formulated in a particular choice of gauge, which causes particular complications when localized-orbital bases or non-local pseudopotentials are used. The simplest quantity to consider is the magnetic susceptibility, the analogue of the electric susceptibility. The coupling in the Hamiltonian can be expressed with the vector potential $\boldsymbol{A}$, field $\boldsymbol{B}=\nabla \times \boldsymbol{A}$, and spin magnetic moment $g \mu_{\mathrm{B}} \cdot \boldsymbol{S}$ ( $\mu_{\mathrm{B}}$ is the Bohr magneton), as

$$
\hat{H}=\frac{1}{2}\left(\boldsymbol{p}+\frac{1}{c} \boldsymbol{A}\right)^{2}+V+g \mu_{\mathrm{B}} \boldsymbol{S} \cdot \boldsymbol{B}=\hat{H}^{(0)}+\frac{1}{c} \boldsymbol{p} \cdot \boldsymbol{A}+\frac{A^{2}}{2 c^{2}}+g \mu_{\mathrm{B}} \boldsymbol{S} \cdot \boldsymbol{B}
$$

The three perturbations are respectively the orbital paramagnetic, (orbital) diamagnetic, and spin (paramagnetic) contributions. With a particular choice of gauge, the first term can also be written as $\boldsymbol{r} \times \boldsymbol{p} \cdot \boldsymbol{B}=\boldsymbol{L} \cdot \boldsymbol{B}$. In spin-unpolarized systems, the spin susceptibility is zero, so just the orbital perturbation is needed. The Sternheimer equation has been used with this perturbation to calculate static susceptibilities for boron fullerene molecules [Botti 2009]. There is actually the advantage, compared to electric perturbations, that to the first-order response of the density is required to be zero if the ground state has time-reversal symmetry, which is the case in the absence of spin-polarization or magnetic fields, so that the magnetic Sternheimer equation does not require self-consistency [Mauri 1996a].

To compute magnetic susceptibilities in solids, we have the same problem in solids as for electric perturbations that the position operator is not periodic, which can similarly be handled either by taking the $\boldsymbol{q} \rightarrow 0$ limit or by the quantum theory of polarization. The $\boldsymbol{q} \rightarrow 0$ approach has been used for 
susceptibilities in crystals [Mauri 1996a]. It has also been used for chemical shifts in nuclear magnetic resonance (NMR) [Mauri 1996b, Pickard 2001], which are ratios between the external field and the environment-dependent screened field at the position of the nuclei. The $g$-tensor of electron paramagnetic resonance (EPR), describing the direction-dependent spin susceptibility, has been calculated by a similar approach for radicals and defects, including spin-orbit and hyperfine effects [Pickard 2002]. The $J$ coupling between nuclear spins in NMR can also be computed by the Sternheimer equation, via the magnetic field induced at one nucleus by the field of another [Joyce 2007]. Susceptibilities can also be studied by applying finite magnetic fields, but in the presence of non-local pseudopotentials the coupling in the Hamiltonian generates additional terms beyond those above to satisfy gauge-invariance, as can be handled with the ICL [Ismail-Beigi 2001] or GIPAW methods [Pickard 2003]. When using pseudopotentials, it is important to note that core susceptibilities may be significant, unlike the electric case; they may be computed from separate atomic calculations [Mauri 1996a], or handled via projector-augmented wave (PAW) methods [Pickard 2001]. The susceptibilities of interest are usually static, and the NMR/EPR properties are treated as static since they are measured at radio frequencies.

To study spin waves in metals, dynamical susceptibilities have been calculated with the Sternheimer equation, where peaks in the spin susceptibility $\chi(\boldsymbol{q}, \omega)$ show the magnon band structure [Savrasov 1998]. Spinor wavefunctions are needed to allow spin rotations. Another kind of magnetic response is the spin-triplet optical excitation spectrum, inaccessible by electric perturbations which can only excite singlets. Time-propagation techniques have been used to calculate triplet states by applying an opposite kick to the up and down spins [Oliveira 2008]. A dynamic response that combines electric and magnetic response is circular dichroism, also known as optical rotation, in which a chiral molecule responds differently to left and right circularly polarized light. The rotatory strength as a function of frequency can be studied via the (orbital) magnetic moment induced by an electric field; the reverse is possible but more complicated to implement. These properties have been calculated for organic molecules with both time-propagation and Sternheimer approaches [Yabana 1999, Varsano 2009]. The techniques have been extended to deal with magneto-optical chiral effects and many other mixed electromagnetical response for finite systems [Espinosa-Leal 2011] (the extension for periodic systems is under development [Andrade 2011]).

\subsubsection{Response to structural perturbations}

There is a rich field of study regarding the response to perturbation of ionic positions and lattice parameters. Since it has been reviewed in great detail [Baroni 2001], and is mostly concerned with static properties, we will consider only briefly most of these quantities and focus on those where TDDFT can be used. Forces on the ions and stresses on the unit cell (the diagonal 
part of which is the pressure) can be calculated via the Hellman-Feynman theorem, which routinely done in static DFT for use in structural relaxation. These forces can additionally be used for calculation of dynamical matrices for vibrational properties by means of the frozen-phonon method, in which finite ionic displacements are used. However, only phonons with commensurate wavectors $\boldsymbol{q}$ can be calculated, and large supercells may be required. Using the Sternheimer equation has the great advantage that dynamical matrices at arbitrary $\boldsymbol{q}$ may be calculated with effort comparable to that for zone-center phonons [Baroni 2001]. For displacement of ion $s$ with potential $V_{s}$ in direction $i$, the perturbation is $\partial V_{s} / \partial R_{s i}$.

The dynamical matrix is diagonalized to obtain phonon frequencies and eigenvectors in the harmonic approximation. This information, as function of cell volume, can also be used as input for the "quasi-harmonic approximation" (QHA) which is used for free energies and other thermodynamic information about solids [Wallace 1972, Born 1954, Carrier 2007]. The phonon group velocities can be computed directly as analytic derivatives from the phonon perturbation calculation as well [Gonze 1989]. Going beyond the harmonic approximation, the $2 n+1$ Theorem gives access to anharmonic properties from Sternheimer calculations [Baroni 2001]. Finite-difference calculations have been used to calculate mechanical anharmonicity and electrical anharmonicity (second-order derivatives of force and polarization with respect to ionic displacement) for ionic contributions to the nonlinear susceptibility [Roman 2006]. Anharmonicities are need for phonon linewidths in crystals, as well as to obtain vibrational frequencies in the presence of strong anharmonicity. Sternheimer phonon calculations also give the induced selfconsistent potential, which is used to calculate the electron-phonon matrix elements between electronic states $i$ and $j$ and a phonon of wavevector $\boldsymbol{q}$ and branch $\nu$ :

$$
g_{i j \nu}(\boldsymbol{k}, \boldsymbol{q})=\left\langle\varphi_{i \boldsymbol{k}+\boldsymbol{q}}\left|\frac{d \hat{H}}{d \lambda_{\boldsymbol{q} \nu}}\right| \varphi_{j \boldsymbol{k}}\right\rangle
$$

With Wannier-function-based interpolation schemes, the electron-phonon coupling has been used to calculate the superconducting properties of borondoped diamond [Giustino 2007] and cuprates [Giustino 2008].

Phonons are generally calculated from static response, an adiabatic approximation which is well justified when the phonon frequency is much less than the electronic band gap. For metals however this condition is not satisfied, and the system may not remain in the electronic ground state during phonon oscillation. Truly dynamical, or non-adiabatic, phonon calculations have been done for doped graphene [Lazzeri 2006] and 2D intercalated compounds [Saitta 2008], showing significant corrections in these systems. A TDDFT sum-over-states perturbation expression is used to find the dynamical matrix at a given frequency, and self-consistently iterated until the input and output frequencies coincide. 
Besides these lattice-dynamics methods, another method for vibrational calculations is molecular dynamics. The system is evolved in time at finite temperature, and from the ionic trajectories, velocity autocorrelation functions are calculated, giving a power spectrum of vibrations as a function of frequency [Allen 1989]. Such calculations can be done by empirical methods or ab initio MD, commonly in the DFT-based Car-Parrinello scheme [Car 1985]. In systems such as liquids, the harmonic approximation fails completely and MD must be used to study the vibrational modes [Putrino 2002] and infrared spectra [Silvestrelli 1997]. Recently a new fast Ehrenfest dynamics method has been developed, in which TDDFT is used to propagate the wavefunctions between timesteps. This allows more efficient calculation of vibrational properties of large systems [Alonso 2008, Andrade 2009]; the method will be discussed in detail in . TDDFT has also been used to study coherent excitation of phonons in $\mathrm{Si}$ by light, propagating the electronic system in the presence of an oscillating applied field, and analyzing the induced forces [Shinohara 2010].

Bulk moduli and elastic constants can be calculated from the second derivative of the total energy with respect to pressure or shear, with finite differences [Lam 1981] or the Sternheimer equation [Baroni 1987a, Baroni 2001]. The strain perturbation involves a stretching of both the unit cell and the wavefunctions, and takes the tensorial form [Nielsen 1985]

$$
T_{i j}=\frac{p_{i} p_{j}}{m}-r_{i} \frac{\partial V_{\mathrm{ext}}}{\partial r_{j}}
$$

Second-order elastic coefficients and Grüneisen parameters (variation of phonon frequencies with stress) can also be calculated from the $2 n+1$ Theorem [Gonze 1989]. The chain rule must be used to include ionic as well as electronic contributions.

\subsubsection{Mixed electric and structural response to structural perturbations}

Raman spectroscopy measures vibrational frequencies by the energy gained or lost by a photon, and in the Placzek approximation the intensity of a vibrational peak is proportional to the Raman tensor [Lazzeri 2003], the derivative of the polarizability with respect to ionic displacement:

$$
\frac{\partial^{3} E}{\partial \mathcal{E}_{i} \partial \mathcal{E}_{j} \partial \mathcal{R}_{k s}}=\frac{\partial \alpha_{i j}}{\partial \mathcal{R}_{k s}}
$$

For solids, the susceptibility $\chi$ can be used instead. For off-resonant Raman, i.e., when the incident phonon is not resonant with an electronic excitation of the system, the static polarizability is generally used. There are many ways the response to the various perturbations could be calculated. Commonly finite differences are used for ionic response, with dielectric tensor calculated 
from sum over states [Baroni 1986], finite differences [Roman 2006], or the Sternheimer equation [Umari 2001]. Anharmonic Raman spectra of ice have been calculated with molecular dynamics by a Fourier transform of the autocorrelation function of the dielectric tensor $\epsilon_{\infty}$.

Purely perturbative methods has also been developed. In an earlier approach applied to silica [Lazzeri 2003], the tensor is written as

$$
\frac{\partial^{3} E}{\partial \mathcal{E}_{i} \partial \mathcal{E}_{j} \partial \mathcal{R}_{k s}}=\int \frac{\partial^{2} \rho}{\partial \mathcal{E}_{i} \partial \mathcal{E}_{j}} \frac{d \hat{H}}{d \mathcal{R}_{k s}} d r
$$

The first-order perturbations are calculated by the Sternheimer equation, and the second-order electric derivatives of the density matrix $\rho$ are calculated via the second-order derivatives of the wavefunctions from a selfconsistent sum-over-states expression. The $2 n+1$ Theorem also makes it possible to do the computation from only first-order ionic and electric derivatives [Veithen 2005]. To study resonant Raman spectroscopy, dynamic polarizabilities must be used. This has been done in TDDFT with the complex polarization propagator approach to study the variation of the Raman spectrum with excitation energy for organic molecules [Mohammed 2009]; this method uses a relaxation toward the ground state in the equations of motion to broaden resonances and prevent divergences.

Another mixed response is Born effective charges, which can be used to calculate LO-TO splitting [Ghosez 1998], infrared spectra [Pasquarello 1997], and molecular dipole moments in liquids [Pasquarello 2003]. They are defined by

$$
Z_{s i j}^{*}=\frac{\partial^{2} E}{\partial \mathcal{E}_{i} \partial R_{s j}}=\frac{\partial \mu_{i}}{\partial R_{s j}}=\frac{\partial F_{s j}}{\partial \mathcal{E}_{i}}
$$

Born charges can be evaluated either as the response of the dipole moment (or polarization) in response to ionic displacement, which is natural in the context of a phonon calculation, or from the forces induced by an electric field, from the Sternheimer equation or finite differences [Gonze 1997b].

A related quantity is the piezoelectric tensor $\gamma$, in which ionic displacement is replaced by strain $e$ :

$$
\gamma_{i j k}^{*}=\frac{\partial^{2} E}{\partial \mathcal{E}_{i} \partial e_{j k}}=\frac{\partial \mu_{i}}{\partial e_{j k}}=\frac{\partial T_{j k}}{\partial \mathcal{E}_{i}}
$$

The strain perturbation is not lattice-periodic, so piezoelectric tensors are most easily calculated by the stress $T$ induced by an electric field [de Gironcoli 1989]. Both electronic and ionic contributions must be included.

A quite different quantity is the non-adiabatic coupling (NAC), which is used in molecular dynamics to govern the rate of hopping between the potential energy surfaces of the ground and excited states [Tully 1990]. Going beyond the Born-Oppenheimer approximation, terms appear in the equation of motion containing $\left\langle\Phi_{i}\left|\frac{\partial}{\partial R_{s j}}\right| \Phi_{j}\right\rangle$ (first-order NAC) and $\left\langle\Phi_{i}\left|\frac{\partial^{2}}{\partial R_{s j}^{2}}\right| \Phi_{j}\right\rangle$ 
(second-order NAC) [Hirai 2009], with overlaps between many-body states $i$, $j$ and their derivatives with respect to ionic displacement. The Casida method can be used for first-order NACs, analogously to the calculation of oscillator strengths but where the dipole operator is replaced with the ionic perturbation [Hu 2007]. A time-propagation formulation has also been developed [Baer 2002] for the calculation. The second-order NACs cannot be calculated by these methods, but are negligible in simple cases [Hirai 2009].

\subsubsection{Response to $k \cdot p$ perturbations}

Response to an infinitesimal shift of $k$-point in a solid, often referred to as $\boldsymbol{k} \cdot \boldsymbol{p}$ perturbation theory, can be used to give various properties. These are by necessity static, not dynamic. Since the perturbation is applied to an individual state rather than to the whole system, it does not have an associated density response. With the Hellman-Feynman Theorem, band velocities can be calculated as

$$
v_{i \boldsymbol{k}}=\frac{1}{\hbar} \frac{\partial \varepsilon_{i \boldsymbol{k}}}{\partial \boldsymbol{k}}=\frac{1}{\hbar}\left\langle u_{i \boldsymbol{k}}\left|\frac{\partial H_{\boldsymbol{k}}}{\partial \boldsymbol{k}}\right| u_{i \boldsymbol{k}}\right\rangle
$$

where $u_{i \boldsymbol{k}}$ is the periodic part of the Bloch function and $H_{\boldsymbol{k}}$ is the effective Hamiltonian it satisfies. The perturbation is

$$
\frac{\partial H_{\boldsymbol{k}}}{\partial \boldsymbol{k}}=-i \nabla_{\boldsymbol{k}}+\boldsymbol{k}+\left[V_{\mathrm{ps}}, \boldsymbol{r}\right]
$$

including a contribution from non-local pseudopotentials if they are used [Rohlfing 2000]. Second-order perturbation theory with a sum over states can similarly give effective masses [Cardona 1966, Yu 1999], as frequently used in simple models of band structures and transport in semiconductors. $\boldsymbol{k} \cdot \boldsymbol{p}$ perturbation theory has also been used, in a finite-difference framework, for $q \rightarrow 0$ limits in $G W$ [Hybertsen 1986] and BSE [Rohlfing 2000] calculations. Additionally, $\boldsymbol{k} \cdot \boldsymbol{p}$ perturbations can be used to compute the $\partial / \partial \boldsymbol{k}$ derivatives which are used in response calculations with the quantum theory of polarization [Olevano 1999, Vanderbilt 2006]. It is important, however, to note that perturbation theory cannot be used to compute the polarization itself, because it does not represent a consistent choice of gauge throughout the Brillouin zone [Resta 1994].

\section{Acknowledgments}

DAS acknowledges support from the US National Science Foundation, Grant No. DMR10-1006184 and graduate fellowship. LL and MALM acknowledges support from the French ANR (ANR-08-CEXC8-008-01). AR acknowledges funding by the European Research Council Advanced Grant DYNamo (ERC2010-AdG -Proposal No. 267374) Spanish MICINN (FIS2010-21282-C02-01), ACI-promociona project (ACI2009-1036), "Grupos Consolidados UPV/EHU 
del Gobierno Vasco" (IT-319-07), and the European Community through e-I3 ETSF project (Contract No. 211956). SGL was supported by the Director, Office of Science, Office of Basic Energy Sciences, Materials Sciences and Engineering Division, U.S. Department of Energy under Contract No. DEAC02-05CH11231.

\section{References}

Allen, M. P. and Tildesley, D. J. (1989). Computer Simulation of Liquids. Oxford University Press, USA.

Alonso, J. L., Andrade, X., Echenique, P., Falceto, F., Prada-Gracia, D. and Rubio, A. (2008). 'Efficient Formalism for Large-Scale Ab Initio Molecular Dynamics based on Time-Dependent Density Functional Theory'. Phys. Rev. Lett. 101(9):096403.

Andrade, X., Botti, S., Marques, M. A. L. and Rubio, A. (2007). 'Timedependent density functional theory scheme for efficient calculations of dynamic (hyper)polarizabilities'. J. Chem. Phys. 126(18):184106.

Andrade, X., Castro, A., Zueco, D., Alonso, J. L., Echenique, P., Falceto, F. and Rubio, A. (2009). 'Modified Ehrenfest Formalism for Efficient Large-Scale ab initio Molecular Dynamics'. J. Chem. Theory Comput. 5(4):728-742.

Andrade, X., Yabana, K., and Rubio, A. (2011). 'Time-dependent polarization theory'. (unpublished)

Anisimov, V. I., Zaanen, J. and Andersen, O. K. (1991). 'Band theory and Mott insulators: Hubbard U instead of Stoner I'. Phys. Rev. B 44(3):943-954.

Baer, R. (2002). 'Non-adiabatic couplings by time-dependent density functional theory'. Chem. Phys. Lett. 364(1-2):75 - 79.

Baroni, S., Gironcoli, S. de, Dal Corso, A. and Giannozzi, P. (2001). 'Phonons and related crystal properties from density-functional perturbation theory'. Rev. Mod. Phys. 73(2):515-562.

Baroni, S., Giannozzi, P. and Testa, A. (1987a). 'Elastic Constants of Crystals from Linear-Response Theory'. Phys. Rev. Lett. 59(23):2662-2665.

Baroni, S., Giannozzi, P. and Testa, A. (1987b). 'Green's-function approach to linear response in solids'. Phys. Rev. Lett. 58(18):1861-1864.

Baroni, S. and Resta, R. (1986). 'Ab initio calculation of the low-frequency Raman cross section in silicon'. Phys. Rev. B 33(8):5969-5971.

Bernard, W. and Callen, H. B. (1959). 'Irreversible thermodynamics of nonlinear processes and noise in driven systems'. Rev. Mod. Phys. 31:1017.

Bishop, D. M. (1990). 'Molecular vibrational and rotational motion in static and dynamic electric fields'. Rev. Mod. Phys. 62(2):343-374.

Born, M. and Huang, K. (1954). Dynamical Theory of Crystal Lattices. Oxford University Press, Oxford.

Botti, S., Castro, A., Andrade, X., Rubio, A. and Marques, M. A. L. (2008). 'Cluster-surface and cluster-cluster interactions: Ab initio calculations and modeling of asymptotic van der Waals forces'. Phys. Rev. B 78(3):035333.

Botti, S., Castro, A., Lathiotakis, N. N., Andrade, X. and Marques, M. A. L. (2009). 'Optical and magnetic properties of boron fullerenes'. Phys. Chem. Chem. Phys. 11:4523-4527. 
Car, R. and Parrinello, M. (1985). 'Unified Approach for Molecular Dynamics and Density-Functional Theory'. Phys. Rev. Lett. 55(22):2471-2474.

Cardona, M. and Pollak, F. H. (1966). 'Energy-Band Structure of Germanium and Silicon: The k·p Method'. Phys. Rev. 142(2):530-543.

Carrier, P., Wentzcovitch, R. and Tsuchiya, J. (2007). 'First-principles prediction of crystal structures at high temperatures using the quasiharmonic approximation'. Phys. Rev. B 76(6):064116.

Casida, M. E. (1995). 'Time-dependent density functional response theory for molecules'. In D. P. Chong (ed.), Recent Advances in Density Functional Methods, pp. 155-192. World Scientific, Singapore.

Cococcioni, M. and Gironcoli, S. de (2005). 'Linear response approach to the calculation of the effective interaction parameters in the LDA $+U$ method'. Phys. Rev. B 71(3):035105.

Dal Corso, A. and Mauri, F. (1994). 'Wannier and Bloch orbital computation of the nonlinear susceptibility'. Phys. Rev. B 50(8):5756-5759.

Dal Corso, A., Mauri, F. and Rubio, A. (1996). 'Density-functional theory of the nonlinear optical susceptibility: Application to cubic semiconductors'. Phys. Rev. B 53(23):15638-15642.

Espinosa-Leal, L., Varsano, D. Andrade, X., Di Felice, R. and Rubio, A. (2011). 'Unified real-time real-space description of Magneto Optical response in closedshell molecular systems'. (to be submitted).

de Gironcoli, S. (1995). 'Lattice dynamics of metals from density-functional perturbation theory'. Phys. Rev. B 51(10):6773-6776.

de Gironcoli, S., Baroni, S. and Resta, R. (1989). 'Piezoelectric properties of IIIV semiconductors from first-principles linear-response theory'. Phys. Rev. Lett. 62(24):2853-2856.

Debernardi, A. and Baroni, S. (1994). 'Third-order density-functional perturbation theory: A practical implementation with applications to anharmonic couplings in Si'. Solid State Commun. 91(10):813 - 816.

Gatti, M., Bruneval, F., Olevano, V. and Reining, L. (2007). 'Understanding Correlations in Vanadium Dioxide from First Principles'. Phys. Rev. Lett. 99(26):266402.

Ghosez, P., Michenaud, J.-P. and Gonze, X. (1998). 'Dynamical atomic charges: The case of $\mathrm{ABO}_{3}$ compounds'. Phys. Rev. B 58(10):6224-6240.

Giustino, F., Cohen, M. L. and Louie, S. G. (2008). 'Small phonon contribution to the photoemission kink in the copper oxide superconductors'. Nature 452(7190):975-978.

Giustino, F., Cohen, M. L. and Louie, S. G. (2010). 'GW method with the selfconsistent Sternheimer equation'. Phys. Rev. B 81(11):115105.

Giustino, F., Yates, J. R., Souza, I., Cohen, M. L. and Louie, S. G. (2007). 'ElectronPhonon Interaction via Electronic and Lattice Wannier Functions: Superconductivity in Boron-Doped Diamond Reexamined'. Phys. Rev. Lett. 98(4):047005.

Gonze, X. (1995a). 'Adiabatic density-functional perturbation theory'. Phys. Rev. A 52(2):1096-1114.

Gonze, X. (1995b). 'Perturbation expansion of variational principles at arbitrary order'. Phys. Rev. A 52(2):1086-1095.

Gonze, X. (1997a). 'First-principles responses of solids to atomic displacements and homogeneous electric fields: Implementation of a conjugate-gradient algorithm'. Phys. Rev. B 55(16):10337-10354. 
Gonze, X. and Lee, C. (1997b). 'Dynamical matrices, Born effective charges, dielectric permittivity tensors, and interatomic force constants from density-functional perturbation theory'. Phys. Rev. B 55(16):10355-10368.

Gonze, X. and Vigneron, J.-P. (1989). 'Density-functional approach to nonlinearresponse coefficients of solids'. Phys. Rev. B 39(18):13120-13128.

Gross, E. K. U. and Kohn, W. (1985). 'Local density-functional theory of frequencydependent linear response'. Phys. Rev. Lett. 55:2850.

Guan, J., Casida, M. E. and Salahub, D. R. (2000). 'Time-dependent densityfunctional theory investigation of excitation spectra of open-shell molecules'. $J$. Molec. Structure (Theochem) 527:229.

Hedin, L. and Lundqvist, S. (1969). 'Effects of electron-electron and electronphonon interactions on the one-electron states of solids'. Solid State Physics 23:1.

Hirai, H. and Sugino, O. (2009). 'A time-dependent density-functional approach to nonadiabatic electron-nucleus dynamics: formulation and photochemical application'. Phys. Chem. Chem. Phys. 11:4570-4578.

$\mathrm{Hu}, \mathrm{C}$., Hirai, H. and Sugino, O. (2007). 'Nonadiabatic couplings from timedependent density functional theory: Formulation in the Casida formalism and practical scheme within modified linear response'. J. Chem. Phys. 127(6):064103.

Hübener, H., Luppi, E. and Véniard, V. (2010). 'Ab initio calculation of second harmonic generation in solids'. phys. stat. sol. (b) 247:1984-1991.

Hybertsen, M. S. and Louie, S. G. (1986). 'Electron correlation in semiconductors and insulators: Band gaps and quasiparticle energies'. Phys. Rev. B 34(8):53905413 .

Hybertsen, M. S. and Louie, S. G. (1987). 'Ab initio static dielectric matrices from the density-functional approach. I. Formulation and application to semiconductors and insulators'. Phys. Rev. B 35(11):5586.

Ismail-Beigi, S., Chang, E. K. and Louie, S. G. (2001). 'Coupling of Nonlocal Potentials to Electromagnetic Fields'. Phys. Rev. Lett. 87(8):087402.

Jamorski, C., Casida, M. E. and Salahub, D. R. (1996). 'Dynamic polarizabilities and excitation spectra from a molecular implementation of time-dependent density-functional response theory: $\mathrm{N}_{2}$ as a case study'. J. Chem. Phys. 104(13):5134-5147.

Joyce, S. A., Yates, J. R., Pickard, C. J. and Mauri, F. (2007). 'A first principles theory of nuclear magnetic resonance J-coupling in solid-state systems'. J. Chem. Phys. 127(20):204107.

Lam, Pui K. and Cohen, Marvin L. (1981). 'Ab initio calculation of the static structural properties of Al'. Phys. Rev. B 24(8):4224-4229.

Langhoff, P. W., Epstein, S. T. and Karplus, M. (1972). 'Aspects of TimeDependent Perturbation Theory'. Rev. Mod. Phys. 44(3):602-644.

Lazzeri, M. and Mauri, F. (2003). 'First-Principles Calculation of Vibrational Raman Spectra in Large Systems: Signature of Small Rings in Crystalline $\mathrm{SiO}_{2}$ '. Phys. Rev. Lett. 90(3):036401.

Lazzeri, M. and Mauri, F. (2006). 'Nonadiabatic Kohn Anomaly in a Doped Graphene Monolayer'. Phys. Rev. Lett. 97(26):266407.

Levine, Z. H. and Allan, D. C. (1989). 'Linear optical response in silicon and germanium including self-energy effects'. Phys. Rev. Lett. 63(16):1719-1722.

Marini, A., Del Sole, R., and Rubio, A. (2006). 'Optical Properties of Solids and Nanostructures from a Many-Body $f_{\mathrm{xc}}$ Kernel'. In M. A. L. Marques, C. A. 
Ullrich, F. Nogueira, A. Rubio, K. Burke, \& E. K. U. Gross (eds.), Time-dependent density functional theory, chap. 20, pp. 301-316. Springer, Berlin Heidelberg.

Marques, M. A. L., Castro, A., Malloci, G., Mulas, G. and Botti, S. (2007). 'Efficient calculation of van der Waals dispersion coefficients with time-dependent density functional theory in real time: Application to polycyclic aromatic hydrocarbons'. J. Chem. Phys. 127(1):014107.

Marques, M. A. L. and Rubio, A. (2006). 'Time Versus Frequency Space Techniques'. In M. A. L. Marques, C. A. Ullrich, F. Nogueira, A. Rubio, K. Burke, \& E. K. U. Gross (eds.), Time-dependent density functional theory, chap. 15, pp. 227-240. Springer, Berlin Heidelberg.

Mauri, F. and Louie, S. G. (1996a). 'Magnetic Susceptibility of Insulators from First Principles'. Phys. Rev. Lett. 76(22):4246-4249.

Mauri, F., Pfrommer, B. G. and Louie, S. G. (1996b). 'Ab Initio Theory of NMR Chemical Shifts in Solids and Liquids'. Phys. Rev. Lett. 77(26):5300-5303.

Mohammed, A., Agren, H. and Norman, P. (2009). 'Time-dependent density functional theory for resonant properties: resonance enhanced Raman scattering from the complex electric-dipole polarizability'. Phys. Chem. Chem. Phys. 11:45394548 .

Nielsen, O. H. and Martin, R. M. (1985). 'Quantum-mechanical theory of stress and force'. Phys. Rev. B 32(6):3780-3791.

Olevano, V., Palummo, M., Onida, G. and Sole, R. D. (1999). 'Exchange and correlation effects beyond the LDA on the dielectric function of silicon'. Phys. Rev. B 60(20):14224-14233.

Oliveira, M. J. T., Castro, A., Marques, M. A. L. and Rubio, A. (2008). 'On the Use of Neumann's Principle for the Calculation of the Polarizability Tensor of Nanostructures'. J. Nanosci. Nanotechnol. 8(7):3392-3398.

Onida, G., Reining, L. and Rubio, A. (2002). 'Electronic excitations: densityfunctional versus many-body Green's-function approaches'. Rev. Mod. Phys. 74(2):601-659.

Pasquarello, A. and Car, R. (1997). 'Dynamical Charge Tensors and Infrared Spectrum of Amorphous $\mathrm{SiO}_{2}$ '. Phys. Rev. Lett. 79(9):1766-1769.

Pasquarello, A. and Resta, R. (2003). 'Dynamical monopoles and dipoles in a condensed molecular system: The case of liquid water'. Phys. Rev. B 68(17):174302.

Petersilka, M., Gossmann, U. J. and Gross, E. K. U. (1996). 'Excitation energies from time-dependent density-functional theory'. Phys. Rev. Lett. 76:1212.

Peterson, R. L. (1967). 'Formal theory of nonlinear response'. Rev. Mod. Phys. 39:69.

Pickard, C. J. and Mauri, F. (2001). 'All-electron magnetic response with pseudopotentials: NMR chemical shifts'. Phys. Rev. B 63(24):245101.

Pickard, C. J. and Mauri, F. (2002). 'First-Principles Theory of the EPR $g$ Tensor in Solids: Defects in Quartz'. Phys. Rev. Lett. 88(8):086403.

Pickard, C. J. and Mauri, F. (2003). 'Nonlocal Pseudopotentials and Magnetic Fields'. Phys. Rev. Lett. 91(19):196401.

Putrino, A. and Parrinello, M. (2002). 'Anharmonic Raman Spectra in HighPressure Ice from Ab Initio Simulations'. Phys. Rev. Lett. 88(17):176401.

Reining, L., Olevano, V., Rubio, A. and Onida, G. (2002). 'Excitonic Effects in Solids Described by Time-Dependent Density-Functional Theory'. Phys. Rev. Lett. 88(6):066404.

Resta, R. (1994). 'Macroscopic polarization in crystalline dielectrics: the geometric phase approach'. Rev. Mod. Phys. 66(3):899-915. 
Rohlfing, M. and Louie, S. G. (2000). 'Electron-hole excitations and optical spectra from first principles'. Phys. Rev. B 62(8):4927-4944.

Roman, E., Yates, J. R., Veithen, M., Vanderbilt, D. and Souza, I. (2006). 'Ab initio study of the nonlinear optics of III-V semiconductors in the terahertz regime'. Phys. Rev. B 74(24):245204.

Sagmeister, S. and Ambrosch-Draxl, C. (2009). 'Time-dependent density functional theory versus Bethe-Salpeter equation: an all-electron study'. Phys. Chem. Chem. Phys. 11(22):4451-4457.

Saitta, A. M., Lazzeri, M., Calandra, M. and Mauri, F. (2008). 'Giant Nonadiabatic Effects in Layer Metals: Raman Spectra of Intercalated Graphite Explained'. Phys. Rev. Lett. 100(22):226401.

Savrasov, S. Y. (1998). 'Linear Response Calculations of Spin Fluctuations'. Phys. Rev. Lett. 81(12):2570-2573.

Senatore, G. and Subbaswamy, K. R. (1987). 'Nonlinear response of closed-shell atoms in the density-functional formalism'. Phys. Rev. A 35(6):2440.

Shen, Y. R. (1984). The principles of nonlinear optics. John Wiley and Sons, New York.

Shinohara, Y., Yabana, K., Kawashita, Y., Iwata, J.-I., Otobe, T. and Bertsch, G. F. (2010). 'Coherent phonon generation in time-dependent density functional theory'. Phys. Rev. B 82(15):155110.

Silvestrelli, P. L., Bernasconi, M. and Parrinello, M. (1997). 'Ab initio infrared spectrum of liquid water'. Chem. Phys. Lett. 277(5-6):478 - 482.

Souza, I., Íñiguez, J. and Vanderbilt, D. (2002). 'First-Principles Approach to Insulators in Finite Electric Fields'. Phys. Rev. Lett. 89(11):117602.

Sternheimer, R. M. (1954). 'Electronic Polarizabilities of Ions from the HartreeFock Wave Functions'. Phys. Rev. 96(4):951-968.

Strubbe, D. A., Andrade, X., Rubio, A. and Louie, S. G. (2011a). 'The timedependent second-order Sternheimer equation'. in preparation.

Strubbe, D. A. and Louie, S. G. (2011b). 'Density-functional perturbation theory with arbitrary fractional occupations'. in preparation .

Takimoto, Y., Vila, F. D. and Rehr, J. J. (2007). 'Real-time time-dependent density functional theory approach for frequency-dependent nonlinear optical response in photonic molecules'. J. Chem. Phys. 127(15):154114.

Tavernelli, I. (2006). 'Electronic density response of liquid water using timedependent density functional theory'. Phys. Rev. B 73(9):094204.

Tiago, M. L. and Chelikowsky, J. R. (2005). 'First-principles GW-BSE excitations in organic molecules'. Solid State Commun. 136(6):333 - 337.

Tully, J. C. (1990). 'Molecular dynamics with electronic transitions'. J. Chem. Phys. 93(2):1061-1071.

Umari, P., Pasquarello, A. and Dal Corso, A. (2001). 'Raman scattering intensities in $\alpha$-quartz: A first-principles investigation'. Phys. Rev. B 63(9):094305.

Vanderbilt, D. and Resta, R. (2006). 'Quantum electrostatics of insulators: polarization, Wannier functions, and electric fields'. In S. G. Louie \& M. L. Cohen (eds.), Conceptual Foundations of Materials, pp. 139-164. Elsevier, Amsterdam.

Varsano, D., Espinosa-Leal, L. A., Andrade, X., Marques, M. A. L., Felice, R. di and Rubio, A. (2009). 'Towards a gauge invariant method for molecular chiroptical properties in TDDFT'. Phys. Chem. Chem. Phys. 11(22):4481-4489.

Veithen, M., Gonze, X. and Ghosez, P. (2005). 'Nonlinear optical susceptibilities, Raman efficiencies, and electro-optic tensors from first-principles density functional perturbation theory'. Phys. Rev. B 71(12):125107. 
Vila, F. D., Strubbe, D. A., Takimoto, Y., Andrade, X., Rubio, A., Louie, S. G. and Rehr, J. J. (2010). 'Basis set effects on the hyperpolarizability of $\mathrm{CHCl}_{3}$ : Gaussian-type orbitals, numerical basis sets and real-space grids'. J. Chem. Phys. 133(3):034111.

Walker, B., Saitta, A. M., Gebauer, R. and Baroni, S. (2006). 'Efficient Approach to Time-Dependent Density-Functional Perturbation Theory for Optical Spectroscopy'. Phys. Rev. Lett. 96(11):113001.

Wallace, D. C. (1972). Thermodynamics of Crystals. John Wiley and Sons, New York and London.

Willetts, A., Rice, J. E., Burland, D. M. and Shelton, D. P. (1992). 'Problems in the comparison of theoretical and experimental hyperpolarizabilities'. J. Chem. Phys. 97(10):7590-7599.

Yabana, K. and Bertsch, G. F. (1996). 'Time-dependent local-density approximation in real time'. Phys. Rev. B 54(7):4484-4487.

Yabana, K. and Bertsch, G. F. (1999). 'Application of the time-dependent local density approximation to optical activity'. Phys. Rev. A 60(2):1271-1279.

Yu, P. Y. and Cardona, M. (1999). Fundamentals of Semiconductors. Springer, Berlin, 2 edn. 